Article

\title{
Rovibrationally Resolved Time-Dependent Collisional-Radiative Model of Molecular Hydrogen and Its Application to a Fusion Detached Plasma
}

\author{
Keiji Sawada ${ }^{1, *}$ and Motoshi Goto ${ }^{2}$ \\ 1 Faculty of Engineering, Shinshu University, 4-17-1 Wakasato, Nagano 380-8553, Japan \\ 2 National Institute for Fusion Science, 322-6 Oroshi-Cho, Toki 509-5292, Japan; goto@nifs.ac.jp \\ * Correspondence: ksawada@shinshu-u.ac.jp \\ Academic Editors: Bastiaan J. Braams, Xavier Urbain, Detlev Reiter and Viatcheslav Kokoouline \\ Received: 30 June 2016; Accepted: 8 November 2016; Published: 20 December 2016
}

\begin{abstract}
A novel rovibrationally resolved collisional-radiative model of molecular hydrogen that includes 4,133 rovibrational levels for electronic states whose united atom principal quantum number is below six is developed. The rovibrational $X^{1} \Sigma_{g}^{+}$population distribution in a SlimCS fusion demo detached divertor plasma is investigated by solving the model time dependently with an initial $300 \mathrm{~K}$ Boltzmann distribution. The effective reaction rate coefficients of molecular assisted recombination and of other processes in which atomic hydrogen is produced are calculated using the obtained time-dependent population distribution.
\end{abstract}

Keywords: collisional-radiative model; molecular hydrogen; vibrational; rotational; population; time-dependent solution

\section{Introduction}

The collisional-radiative model (CR model) [1] provides population distributions of atoms and molecules. Using this model, the effective reaction rate coefficients of various atomic and molecular processes including the contributions of the excited states can be calculated based on the population distribution. Emission intensities of atoms and molecules are also calculated using the population distribution.

We have been developing a CR model of molecular hydrogen, $\mathrm{H}_{2}$. Previously, a CR model for molecular hydrogen in which only the electronic states are considered was constructed by Sawada and Fujimoto [2]. This model was used to calculate effective reaction rate coefficients of dissociation and ionization of the molecule in plasmas. This CR model was extended by Greenland and Reiter [3] and Fantz and Wündrlich [4], and the rate coefficients calculated by these models were included in the neutral transport code EIRENE [5].

Molecular-assisted recombination (MAR) was then proposed to understand the detached recombining plasmas in fusion edge plasmas [6]. MAR comprises two series of processes. The first starts with the production of $\mathrm{H}_{2}^{+}$from the collision of $\mathrm{H}_{2}$ and $\mathrm{H}^{+}$, and the second starts with the dissociative attachment of electrons. To evaluate the contribution of the molecular vibrational distribution to the rate coefficient of MAR, we constructed a model in which the vibrational states were included in addition to the electronic states [7].

It has recently been recognized that the production of state-resolved cross-sections is essential for the detailed analysis of fusion divertor plasmas [8]. In this study, we develop a CR model in which the electronic, vibrational and rotational states are considered in order to model molecular processes whose cross-sections strongly depend on the initial vibrational and rotational states, e.g., the dissociative attachment of electrons in MAR. 
As for the emission spectroscopy of molecular hydrogen, a corona model [1] for the Fulcher transition $\left(d^{3} \Pi_{u} \rightarrow a^{3} \Sigma_{g}^{+}\right)$that includes vibrational states was constructed to determine the vibrational temperature in the electronic ground state $X^{1} \Sigma_{g}^{+}$[9]. Although the model was developed to also include rotational states in order to determine both rotational and vibrational temperatures in $X^{1} \Sigma_{g}^{+}[10]$, corona models may not be suited to high electron density fusion detached plasmas because they neglect electron impact transition among the excited states. To describe high electron density plasmas, a rovibrationally-resolved CR model is necessary. In addition to the Fulcher band, this model provides emission line intensities for many bands, which may be used to determine the electron temperature and density as in helium atom spectroscopy [11] and to determine the rovibrational population distribution in the $X^{1} \Sigma_{g}^{+}$without assuming the vibrational and rotational Boltzmann distributions.

The purpose of this paper is to introduce a newly-developed CR model and to provide examples of its calculation. In this study, we investigated the rovibrational population distribution in a fusion detached divertor plasma. Because the quasi-steady-state solution [1] for rovibrational states in $X^{1} \Sigma_{g}^{+}$ cannot be applied to the rate equations in the CR model owing to the large population relaxation time, we solved the rate equations time dependently for these states.

In this paper, we first introduce our newly-developed state-resolved CR model of molecular hydrogen and then list considered energy levels and relevant data as well as spontaneous transition probabilities, cross-sections and rate coefficients used in the model. Finally, we report on the modeling of a SlimCS fusion demo detached divertor plasma [12,13] in which effective reaction rate coefficients including MAR are calculated using the rovibrational population distribution. An example of emission spectra is also shown.

\section{Collisional-Radiative Model}

\subsection{Framework of the Collisional-Radiative Model}

To provide a framework of the CR model itself, we describe a model of atomic hydrogen $[1,14,15]$ for simplicity. Here we assume that only the atomic hydrogen, electrons and protons exist in a plasma. The CR model gives the population of an excited state $p, n(p)$. The temporal variation of the population density of an excited state $p$ can be described by a rate equation as:

$$
\begin{aligned}
d n(p) / d t= & \sum_{q<p} C(q, p) n_{\mathrm{e}} n(q)+\sum_{q>p}\left[F(q, p) n_{\mathrm{e}}+A(q, p)\right] n(q)+\alpha(p) n_{\mathrm{H}^{+}} n_{\mathrm{e}}^{2}+\beta(p) n_{\mathrm{H}^{+}} n_{\mathrm{e}} \\
& -\left\{\left[\sum_{q<p} F(p, q)+\sum_{q>p} C(p, q)+S(p)\right] n_{\mathrm{e}}+\sum_{q<p} A(p, q)\right\} n(p)
\end{aligned}
$$

where $n_{e}$ and $n_{\mathrm{H}^{+}}$are the electron and proton densities, respectively, $C(p, q)$ is the excitation rate coefficient for electron collisions from state $p$ to $q$ and $F(q, p)$ is the inverse de-excitation rate coefficient. $A(p, q)$ is the spontaneous transition probability from $p$ to $q . S(p)$ is the ionization rate coefficient for state $p$, and $\alpha(p)$ and $\beta(p)$ are three-body and radiative recombination rate coefficients, respectively. Each rate coefficient is a function of the electron temperature $T_{\mathrm{e}}$.

In many cases, the quasi-steady-state solution (QSS) [1] can be used to approximate the left-hand side of Equation (1) as zero for all states except the ground state:

$$
\frac{d}{d t} n(p)=0
$$

By solving Equation (1) with Equation (2), this is a set of coupled linear equations, the population density of an excited state $p$ in the form of:

$$
n(p)=R_{0}(p) n_{\mathrm{H}^{+}} n_{\mathrm{e}}+R_{1}(p) n(1) n_{\mathrm{e}}
$$

is obtained, where $R_{0}(p)$ and $R_{1}(p)$ are the population coefficients, each of which is a function of $n_{\mathrm{e}}$ and $T_{\mathrm{e}}$. The first and the second terms are referred to as the recombining and the ionizing components, respectively [1]. 
The population of the ground state $n(1)$ should be solved time dependently using the population coefficients for the excited state in Equation (3) [15]:

$$
\begin{aligned}
\frac{d}{d t} n(1)= & {\left[\sum_{p>1}\left(A(p, 1)+F(p, 1) n_{\mathrm{e}}\right)\left(R_{0}(p) n_{\mathrm{H}^{+}} n_{\mathrm{e}}+R_{1}(p) n(1) n_{\mathrm{e}}\right)+\alpha(1) n_{\mathrm{H}^{+}} n_{\mathrm{e}}^{2}+\beta(1) n_{\mathrm{H}^{+}} n_{\mathrm{e}}\right] } \\
- & {\left[\sum_{p>1} C(p, 1) n(1) n_{\mathrm{e}}+S(1) n(1) n_{\mathrm{e}}\right], }
\end{aligned}
$$

where the first and the second terms in the right-hand side of Equation (4) denote inflow to and outflow from the ground state, respectively. Equation (4) is simply rewritten as

$$
\frac{d}{d t} n(1)=\alpha_{\mathrm{CR}} n_{\mathrm{H}^{+}} n_{\mathrm{e}}-S_{\mathrm{CR}} n(1) n_{\mathrm{e}}
$$

by defining the effective recombination and ionization rate coefficients, $\alpha_{\mathrm{CR}}$ and $S_{\mathrm{CR}}$, respectively. Both $\alpha_{\mathrm{CR}}$ and $S_{\mathrm{CR}}$ are functions of $n_{\mathrm{e}}$ and $T_{\mathrm{e}}$.

We define $\tau(p)$ as the reciprocal of the outflow rate in Equation (1):

$$
\tau(p)=\left\{\left[\sum_{q<p} F(p, q)+\sum_{q>p} C(p, q)+S(p)\right] n_{\mathrm{e}}+\sum_{q<p} A(p, q)\right\}^{-1} .
$$

The value of $\tau(p)$ gives the relaxation time after the inflow to the level $p$ becomes steady. The relaxation time of all excited levels is given approximately by the maximum value of $\tau(p)$ in the excited levels [15], which we define as $\tau_{\max }$. In applying the QSS for the excited states, the environment of the excited states, $n(1), n_{\mathrm{e}}, n_{\mathrm{H}^{+}}$, and $T_{\mathrm{e}}$, should be steady on the time scale of $\tau_{\max }$. In many cases, this condition is satisfied; the ground state $\tau(1)$ is much larger than $\tau_{\max }$ because there is no spontaneous transition. For more details, see [15].

\subsection{Collisional-Radiative Model for Molecular Hydrogen}

In the case of molecular hydrogen, the values of $\tau(p)$ for the rovibrational states in the electronic ground state $X^{1} \Sigma_{g}^{+}$are nearly the same, and the populations of all of these states should therefore be solved time dependently. In this study, the populations of $c^{3} \Pi_{u}^{-}(v=0)$, which is known as the metastable state, and the $a^{3} \Sigma_{g}^{+}$, which couples strongly to the $c^{3} \Pi_{u}^{-}(v=0)$ state, are solved time dependently in addition to the population of $X^{1} \Sigma_{g}^{+}$. In the application of this study, as shown later, we deal with the population of molecular hydrogen originating from the electron impact excitation of the ground state $X^{1} \Sigma_{g}^{+}$, which corresponds to the ionizing plasma component in Equation (3).

\section{Elementary Processes}

Here, we introduce elementary processes included in the molecular hydrogen CR model. The projectiles considered are listed in Table 1.

Table 1. Target and projectiles in this study.

\begin{tabular}{cc}
\hline Target & Projectile \\
\hline $\mathrm{H}_{2}$ & $\mathrm{e}, \mathrm{H}^{+}, \mathrm{H}_{1} \mathrm{H}_{2}$ \\
\hline
\end{tabular}

In the following, references are given for the data used; for discussion of their respective reliabilities, the original papers should be consulted. We will provide comments regarding data with large uncertainty.

\subsection{Energy Levels of $\mathrm{H}_{2}$}

In this study, the energy levels of molecular hydrogen are labeled $e, v, N$, and $J$ based on Hund's coupling Case (b) $[16,17]$. Here, $e$ is the set of quantum numbers characterizing the electronic state; $v$ is 
the vibrational quantum number; $N$ is the rotational quantum number of the total angular momentum excluding electron and nuclear spins; and $J$ is the quantum number of the total angular momentum excluding nuclear spin. For the statistical weights and allowed transition in the rotational levels, see [16]. For electronic states with $\Lambda>0$, the two levels associated with the two possible values $\pm|\Lambda|$ are considered. Here, $\Lambda$ is a projection of total electronic orbital angular momentum about the internuclear axis [16].

The energy level data are taken from [18-20]. Table 2 lists references adopted in the present model. For triplet data in [18], $149.6 \mathrm{~cm}^{-1}$ is subtracted according to [21,22]. Figure 1 shows the electronic states whose energy levels are given in [18-20]. 4,133 rovibrational states with the united atom principal quantum number $n \leq 6$ is included in the present model.

Table 2. References for energy levels and potentials.

\begin{tabular}{cccc}
\hline State & United Atom & Energy Level & Potential \\
\hline$X^{1} \Sigma_{g}^{+}$ & $(1 s \sigma)^{2}$ & {$[19]$} & {$[23]$} \\
$E F^{1} \Sigma_{g}^{+}$ & $1 s \sigma 2 p \sigma+(2 p \sigma)^{2}$ & {$[20]$} & {$[24]$} \\
$H \bar{H}^{1} \Sigma_{g}^{+}$ & $1 s \sigma 3 s \sigma$ & {$[20]$} & {$[25]$} \\
$O^{1} \Sigma_{g}^{+}$ & $1 s \sigma 4 s \sigma$ & our calculation & {$[25]$} \\
\hline$B^{1} \Sigma_{u}^{+}$ & $1 s \sigma 2 p \sigma$ & {$[20]$} & {$[26]$} \\
$B^{\prime} \Sigma_{u}^{+}$ & $1 s \sigma 3 p \sigma$ & {$[20]$} & {$[26]$} \\
$B^{\prime \prime} \bar{B}^{1} \Sigma_{u}^{+}$ & $1 s \sigma 4 p \sigma$ & our calculation & {$[26]$} \\
\hline$C^{1} \Pi_{u}$ & $1 s \sigma 2 p \pi$ & {$[20]$} & {$[27]$} \\
$D^{1} \Pi_{u}$ & $1 s \sigma 3 p \pi$ & {$[20]$} & {$[27]$} \\
$D^{\prime 1} \Pi_{u}$ & $1 s \sigma 4 p \pi$ & our calculation & {$[27]$} \\
\hline$G K^{1} \Sigma_{g}^{+}$ & $1 s \sigma 3 d \sigma$ & {$[20]$} & {$[25]$} \\
$P^{1} \Sigma_{g}^{+}$ & $1 s \sigma 4 d \sigma$ & {$[18]$} & {$[25]$} \\
\hline$I^{1} \Pi_{g}$ & $1 s \sigma 3 d \pi$ & {$[20]$} & {$[28]$} \\
$R^{1} \Pi_{g}$ & $1 s \sigma 4 d \pi$ & {$[18]$} & {$[28]$} \\
\hline$J^{1} \Delta_{g}$ & $1 s \sigma 3 d \delta$ & {$[20]$} & {$[28]$} \\
$S^{1} \Delta_{g}$ & $1 s \sigma 4 d \delta$ & {$[18]$} & {$[28]$} \\
\hline$V^{1} \Pi_{u}$ & $1 s \sigma 4 f \pi$ & our calculation & {$[27]$} \\
\hline$a^{3} \Sigma_{g}^{+}$ & $1 s \sigma 2 s \sigma$ & {$[18]$} & {$[29]$} \\
$h^{3} \Sigma_{g}^{+}$ & $1 s \sigma 3 s \sigma$ & {$[18]$} & {$[29]$} \\
$4 s^{3} \Sigma_{g}^{+}$ & $1 s \sigma 4 s \sigma$ & {$[18]$} & {$[30]$} \\
\hline$b^{3} \Sigma_{u}^{+}$ & $1 s \sigma 2 p \sigma$ & - & {$[29]$} \\
$e^{3} \Sigma_{u}^{+}$ & $1 s \sigma 3 p \sigma$ & {$[18]$} & {$[29]$} \\
$f^{3} \Sigma_{u}^{+}$ & $1 s \sigma 4 p \sigma$ & {$[18]$} & {$[29]$} \\
\hline$c^{3} \Pi_{u}$ & $1 s \sigma 2 p \pi$ & {$[18]$} & {$[29]$} \\
$d^{3} \Pi_{u}$ & $1 s \sigma 3 p \pi$ & {$[18]$} & {$[29]$} \\
$k^{3} \Pi_{u}$ & $1 s \sigma 4 p \pi$ & {$[18]$} & {$[29]$} \\
\hline$g^{3} \Sigma_{g}^{+}$ & $1 s \sigma 3 d \sigma$ & {$[18]$} & {$[29]$} \\
$p^{3} \Sigma_{g}^{+}$ & $1 s \sigma 4 d \sigma$ & {$[18]$} & {$[30]$} \\
\hline$i^{3} \Pi_{g}$ & $1 s \sigma 3 d \pi$ & {$[18]$} & {$[29]$} \\
$r^{3} \Pi_{g}$ & $1 s \sigma 4 d \pi$ & {$[18]$} & {$[29]$} \\
\hline$j^{3} \Delta_{g}$ & $1 s \sigma 3 d \delta$ & {$[18]$} & {$[28]$} \\
$s^{3} \Delta_{g}$ & $1 s \sigma 4 d \delta$ & {$[18]$} & {$[28]$} \\
\hline$m^{3} \Sigma_{u}^{+}$ & $1 s \sigma 4 f \pi$ & our calculation & {$[31]$} \\
\hline & & & \\
\hline & & $18]$ & \\
\hline
\end{tabular}




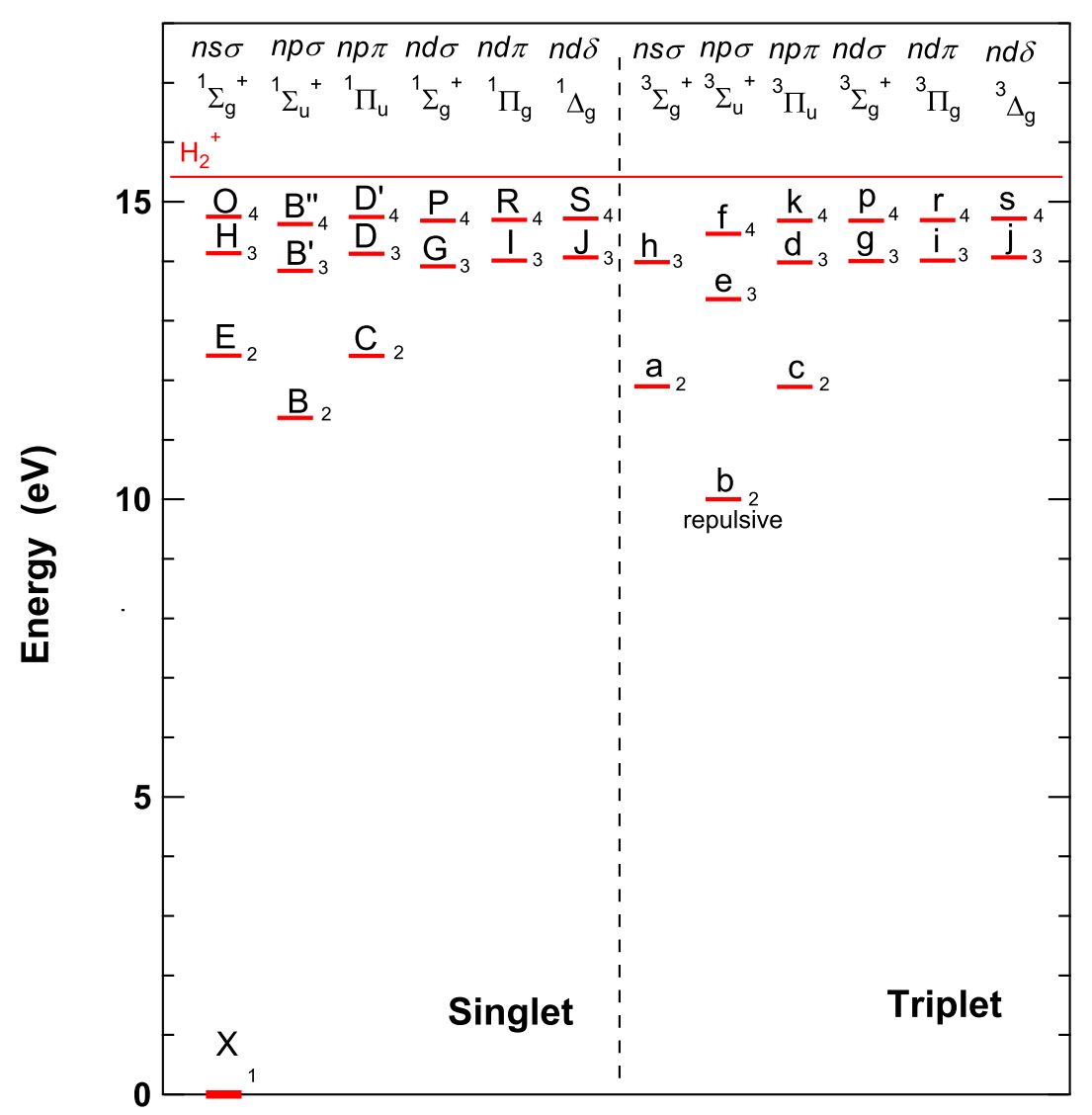

Figure 1. The energy level of the rovibrational ground state for each electronic state in [18-20] is shown. For the repulsive state $b^{3} \Sigma_{u}^{+}$, the energy level at the nuclear distance of the potential minimum of $X^{1} \Sigma_{g}^{+}$ is shown. In the present model, states up to $n=6$ are considered.

We calculate the rovibrational energy levels for which data are not available using the electronic potential as follows.

\subsection{Electronic Potential Data}

A review of electronic state potentials is available in [32], and Table 2 lists the potential references adopted in our model. Figure 2a-c shows the potential curves from the references in Table 2.

To calculate the energy level and the Franck-Condon factor [16], we solve the Schrödinger equation for Hund's (b) case [16]:

$$
-\frac{\hbar^{2}}{2 \mu} \frac{\partial^{2} \psi_{e v N}(R)}{\partial R^{2}}+\left(V(R)+\frac{\hbar^{2}}{2 \mu} \frac{N(N+1)}{R^{2}}\right) \psi_{e v N}(R)=E \psi_{e v N}(R)
$$

where $\psi_{e v N}(R)$ is the wave function. This is solved using the Numerov method [33]. 

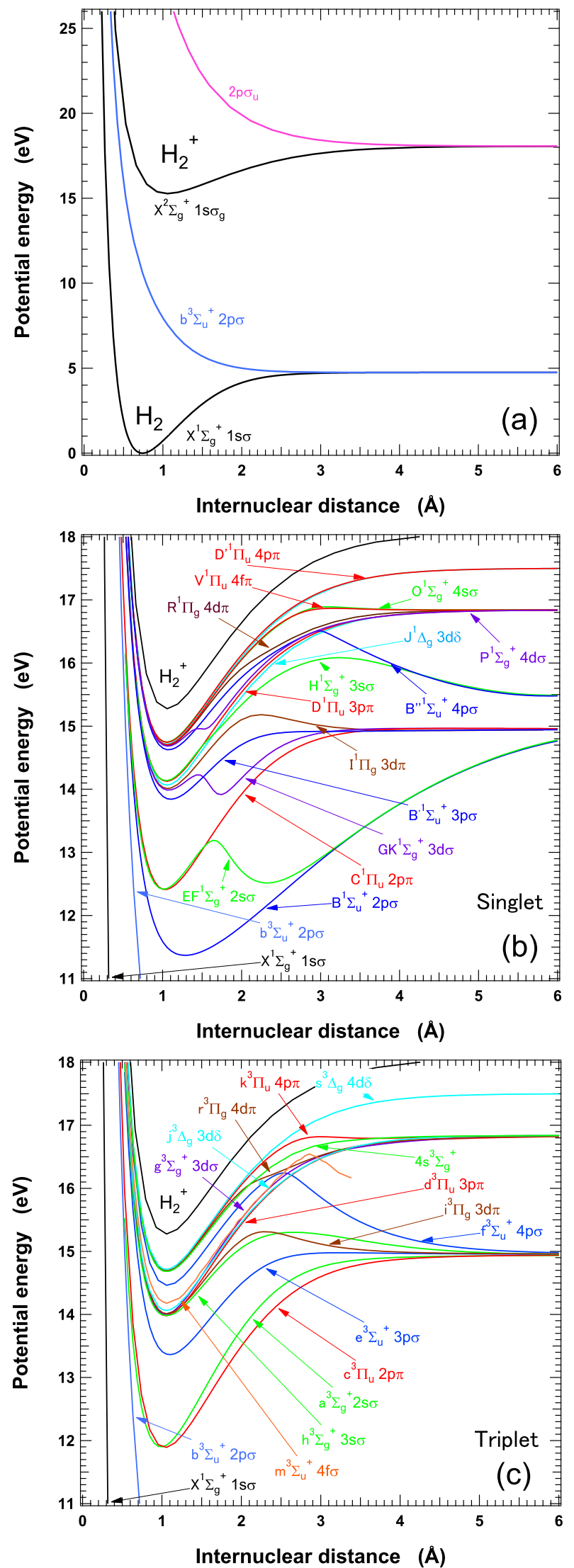

Figure 2. Potential curves. (a) $\mathrm{H}_{2}$ and $\mathrm{H}_{2}^{+}$; (b) singlet; (c) triplet. 


\subsection{Spontaneous Transition and Predissociation}

The bound-bound transition probability data in Table 3 are included in the model. We calculated other transition probabilities using the following equation [34]:

$$
A\left(e v J, e^{\prime} v^{\prime} J^{\prime}\right)=\frac{16 \pi^{3}}{3 \epsilon_{0} h \lambda^{3}} \frac{1}{2 J+1}\left|\int \psi_{e v J}(R) R_{e}(R) \psi_{e^{\prime} v^{\prime} J^{\prime}}(R) d R\right|^{2} S_{J J^{\prime}}
$$

where the labels $e, v, J$ and $e^{\prime}, v^{\prime}, J^{\prime}$ denote the initial and final electronic, vibrational and rotational states, respectively, and $S_{J J^{\prime}}$ is the Hönl-London factor [16], which is given in [35]. In the present model, the transition moments $[25,27,29,36-38]$ in Table 4 are used, while the united helium atom transition moments are used for other transitions.

Table 3. References for the transition probability.

\begin{tabular}{cc}
\hline Transition & Present \\
\hline$I^{1} \Pi_{g} \rightarrow C^{1} \Pi_{u}$ & {$[39]$} \\
$J_{g}^{1} \Delta_{g} \rightarrow C^{1} \Pi_{u}$ & {$[40]$} \\
$B^{1} \Sigma_{u}^{+}, C^{1} \Pi_{u}, B^{\prime 1} \Sigma_{u}^{+}, D^{1} \Pi_{u} \rightarrow X^{1} \Sigma_{g}^{+}$ & {$[41-43]$} \\
\hline$d^{3} \Pi_{u}, e \Sigma_{u}^{+} \rightarrow a^{3} \Sigma_{g}^{+}$ & {$[40]$} \\
$i^{3} \Pi_{g}, j^{3} \Delta_{g} \rightarrow c^{3} \Pi_{u}$ & {$[40]$} \\
$k^{3} \Pi_{u} \rightarrow a^{3} \Sigma_{g}^{+}$ & {$[44]$} \\
\hline
\end{tabular}

Table 4. References for the transition moments.

\begin{tabular}{cc}
\hline Transition & Present \\
\hline$X^{1} \Sigma_{g}^{+} \leftrightarrow C^{1} \Pi_{u}, D^{1} \Pi_{u}, D^{1} \Pi_{u}, V^{1} \Pi_{u}$ & {$[27]$} \\
$X^{1} \Sigma_{g}^{+} \leftrightarrow B^{1} \Sigma_{u}^{+}, B^{\prime 1} \Sigma_{u}^{+}, B^{\prime \prime 1} \Sigma_{u}^{+}, B 4^{1} \Sigma_{u}^{+}, B 5^{1} \Sigma_{u}^{+}, B 6^{1} \Sigma_{u}^{+}$ & {$[36]$} \\
$C^{1} \Pi_{u} \leftrightarrow I^{1} \Pi_{g}, R^{1} \Pi_{g}, J^{1} \Delta_{g}, S^{1} \Delta_{g}$ & {$[37]$} \\
$D^{1} \Pi_{u} \leftrightarrow I^{1} \Pi_{g}, R^{1} \Pi_{g}, J^{1} \Delta_{g}, S^{1} \Delta_{g}$ & {$[37]$} \\
$B^{1} \Sigma_{u}^{+} \leftrightarrow E F^{1} \Sigma_{g}^{+}, H \bar{H}^{1} \Sigma_{g}^{+}, O^{1} \Sigma_{g}^{+}$ & {$[25]$} \\
$B^{\prime 1} \Sigma_{u}^{+} \leftrightarrow E F^{1} \Sigma_{g}^{+}, H \bar{H}^{1} \Sigma_{g}^{+}, O^{1} \Sigma_{g}^{+}$ & {$[25]$} \\
$B^{\prime \prime 1} \Sigma_{u}^{+} \leftrightarrow E F^{1} \Sigma_{g}^{+}, H \bar{H}^{1} \Sigma_{g}^{+}, O^{1} \Sigma_{g}^{+}$ & {$[25]$} \\
$C^{1} \Pi_{u} \leftrightarrow E F^{1} \Sigma_{g}^{+}, H \bar{H}^{1} \Sigma_{g}^{+}, O^{1} \Sigma_{g}^{+}$ & {$[25]$} \\
$D^{1} \Pi_{u} \leftrightarrow E F^{1} \Sigma_{g}^{+}, H \bar{H}^{1} \Sigma_{g}^{+}, O^{1} \Sigma_{g}^{+}$ & {$[25]$} \\
\hline$b^{3} \Sigma_{u}^{+} \leftrightarrow a^{3} \Sigma_{g}^{+}, h^{3} \Sigma_{g}^{+}, g^{3} \Sigma_{g}^{+}, i^{3} \Pi_{g}, r^{3} \Pi_{g}$ & {$[29]$} \\
$c^{3} \Pi_{u} \leftrightarrow a^{3} \Sigma_{g}^{+}, h^{3} \Sigma_{g}^{+}, g^{3} \Sigma_{g}^{+}, i^{3} \Pi_{g}, r^{3} \Pi_{g}$ & {$[29]$} \\
$d^{3} \Pi_{u} \leftrightarrow a^{3} \Sigma_{g}^{+}, h^{3} \Sigma_{g}^{+}, g^{3} \Sigma_{g}^{+}, i^{3} \Pi_{g}, r^{3} \Pi_{g}$ & {$[29]$} \\
$e^{3} \Sigma_{u}^{+} \leftrightarrow a^{3} \Sigma_{g}^{+}, h^{3} \Sigma_{g}^{+}, g^{3} \Sigma_{g}^{+}, i^{3} \Pi_{g}, r^{3} \Pi_{g}$ & {$[29]$} \\
$f^{3} \Sigma_{u}^{+} \leftrightarrow a^{3} \Sigma_{g}^{+}, h^{3} \Sigma_{g}^{+}, g^{3} \Sigma_{g}^{+}, i^{3} \Pi_{g}, r^{3} \Pi_{g}$ & {$[29]$} \\
$k^{3} \Pi_{u} \leftrightarrow a^{3} \Sigma_{g}^{+}, h^{3} \Sigma_{g}^{+}, g^{3} \Sigma_{g}^{+}, i^{3} \Pi_{g}, r^{3} \Pi_{g}$ & {$[29]$} \\
$w^{3} \Pi_{g} \leftrightarrow b^{3} \Sigma_{u}^{+}, g^{3} \Sigma_{g}^{+}, f^{3} \Sigma_{u}^{+}, k^{3} \Pi_{u}$ & {$[38]$} \\
\hline
\end{tabular}

We included the transitions in Table 5, which are accompanied by the continuum emission. The transition probability from a discrete vibrational state $v$ to a continuum vibrational state with wavenumber $k^{\prime}$ is calculated as [45-47]:

$$
A_{v k^{\prime}}=9.23 \times 10^{-12} g v_{v k^{\prime}}^{3}\left|\left\langle\psi_{v}(R)|D(R)| \psi_{k^{\prime}}(R)\right\rangle\right|^{2}
$$

where $R$ is the nuclear distance and $v_{v k^{\prime}}$ is the photon frequency in $\mathrm{cm}^{-1}$. The wave functions $\psi_{v}(R)$ and $\psi_{k^{\prime}}(R)$ pertain to the upper and lower states, respectively. $D(R)$ is the transition moment between the electronic states; $v_{v k^{\prime}}$ is the frequency of the emitted light; and $g$ is the statistical weight. Table 5 
lists references for $D(R)$ used in the present model, and $\psi_{k^{\prime}}(R)$ is calculated by applying the Numerov method [33] to Equation (7), and $\psi_{k^{\prime}}(R)$ is normalized at large $R$ according to [46,47].

Table 5. References for the transition moment of the bound-continuum.

\begin{tabular}{cc}
\hline Transition & Present \\
\hline$B^{1} \Sigma_{u}^{+}, B^{\prime 1} \Sigma_{u}^{+}, B^{\prime \prime 1} \Sigma_{u}^{+} \rightarrow X^{1} \Sigma_{g}^{+}$continuum & {$[36]$} \\
$C^{1} \Pi_{u}, D^{1} \Pi_{u} \rightarrow X^{1} \Sigma_{g}^{+}$continuum & {$[27]$} \\
$a^{3} \Sigma_{g}^{+}, h^{3} \Sigma_{g}^{+}, g^{3} \Sigma_{g}^{+}, i^{3} \Pi_{g}, r^{3} \Pi_{g} \rightarrow b^{3} \Sigma_{u}^{+}$ & {$[29]$} \\
\hline
\end{tabular}

Predissociation lifetimes from the metastable state $c^{3} \Pi_{u}^{-}(v=0)$ to the $b^{3} \Sigma_{u}^{+}$, as given in [48], are included in the model with values on the order of $10^{-3} \mathrm{~s}$.

\subsection{Electron Impact Electronic Excitation}

\subsubsection{Excitation from the Electronic Ground State $X^{1} \Sigma_{g}^{+}$}

Tables 6 and 7 show the references for the electron impact cross-sections from the $X^{1} \Sigma_{g}^{+}$to the electronic excited states. Cross-sections to the $B^{1} \Sigma_{u}^{+}$and $C^{1} \Pi_{u}$ are shown in Figure 3 as an example. Aside from some data from [49], we use semi-empirical data from [50,51], although the reliability of these data has not been closely examined.

Table 6. References for electron impact excitation cross-section from $X^{1} \Sigma_{g}^{+}$to various singlet states.

\begin{tabular}{cccc}
\hline Excited State & $\mathbf{e} \rightarrow \mathbf{e}^{\prime}$ & $\mathbf{e v} \rightarrow \mathbf{e}^{\prime}$ & $\mathbf{e v} \rightarrow \mathbf{e}^{\prime} \mathbf{v}^{\prime}$ \\
\hline$E F^{1} \Sigma_{g}^{+}$ & {$[50]$} & & \\
$H \bar{H}^{1} \Sigma_{g}^{+}$ & {$[50]$} & & \\
$O^{1} \Sigma_{g}^{+}$ & {$[51]$} & & \\
\hline$B^{1} \Sigma_{u}^{+}$ & & & {$[49]$} \\
$B^{\prime} \Sigma_{u}^{+}$ & & {$[49]$} & \\
$B^{\prime \prime} \bar{B}^{1} \Sigma_{u}^{+}$ & & {$[49]$} & \\
\hline$C^{1} \Pi_{u}$ & & & {$[49]$} \\
$D^{1} \Pi_{u}$ & & {$[49]$} & \\
$D^{\prime 1} \Pi_{u}$ & & {$[49]$} & \\
\hline$G K^{1} \Sigma_{g}^{+}$ & {$[50]$} & & \\
$P^{1} \Sigma_{g}^{+}$ & {$[51]$} & & \\
\hline$I^{1} \Pi_{g}$ & {$[50]$} & & \\
$R^{1} \Pi_{g}$ & {$[51]$} & & \\
\hline$J^{1} \Delta_{g}$ & {$[51]$} & & \\
$S^{1} \Delta_{g}$ & {$[51]$} & & \\
\hline$V^{1} \Pi_{u}$ & {$[51]$} & & \\
\hline
\end{tabular}


Table 7. References for electron impact excitation cross-section from $X^{1} \Sigma_{g}^{+}$to various triplet states.

\begin{tabular}{ccc}
\hline Excited State & $\mathbf{e} \rightarrow \mathbf{e}^{\prime}$ & $\mathbf{e v} \rightarrow \mathbf{e}^{\prime}$ \\
\hline$a^{3} \Sigma_{g}^{+}$ & {$[50]$} & \\
$h^{3} \Sigma_{g}^{+}$ & {$[51]$} & \\
$4 s^{3} \Sigma_{g}^{+}$ & {$[51]$} & \\
\hline$b^{3} \Sigma_{u}^{+}$ & & {$[49]$} \\
$e^{3} \Sigma_{u}^{+}$ & {$[50]$} & \\
$f^{3} \Sigma_{u}^{+}$ & {$[51]$} & \\
\hline$c^{3} \Pi_{u}$ & {$[50]$} & \\
$d^{3} \Pi_{u}$ & {$[52]$} & \\
$k^{3} \Pi_{u}$ & {$[51]$} & \\
\hline$g^{3} \Sigma_{g}^{+}$ & {$[51]$} & \\
$p^{3} \Sigma_{g}^{+}$ & {$[51]$} & \\
\hline$i^{3} \Pi_{g}$ & {$[51]$} & \\
$r^{3} \Pi_{g}$ & {$[51]$} & \\
\hline$j^{3} \Delta_{g}$ & {$[51]$} & \\
$s^{3} \Delta_{g}$ & {$[51]$} & \\
\hline$m^{3} \Sigma_{u}^{+}$ & {$[51]$} & \\
\hline
\end{tabular}

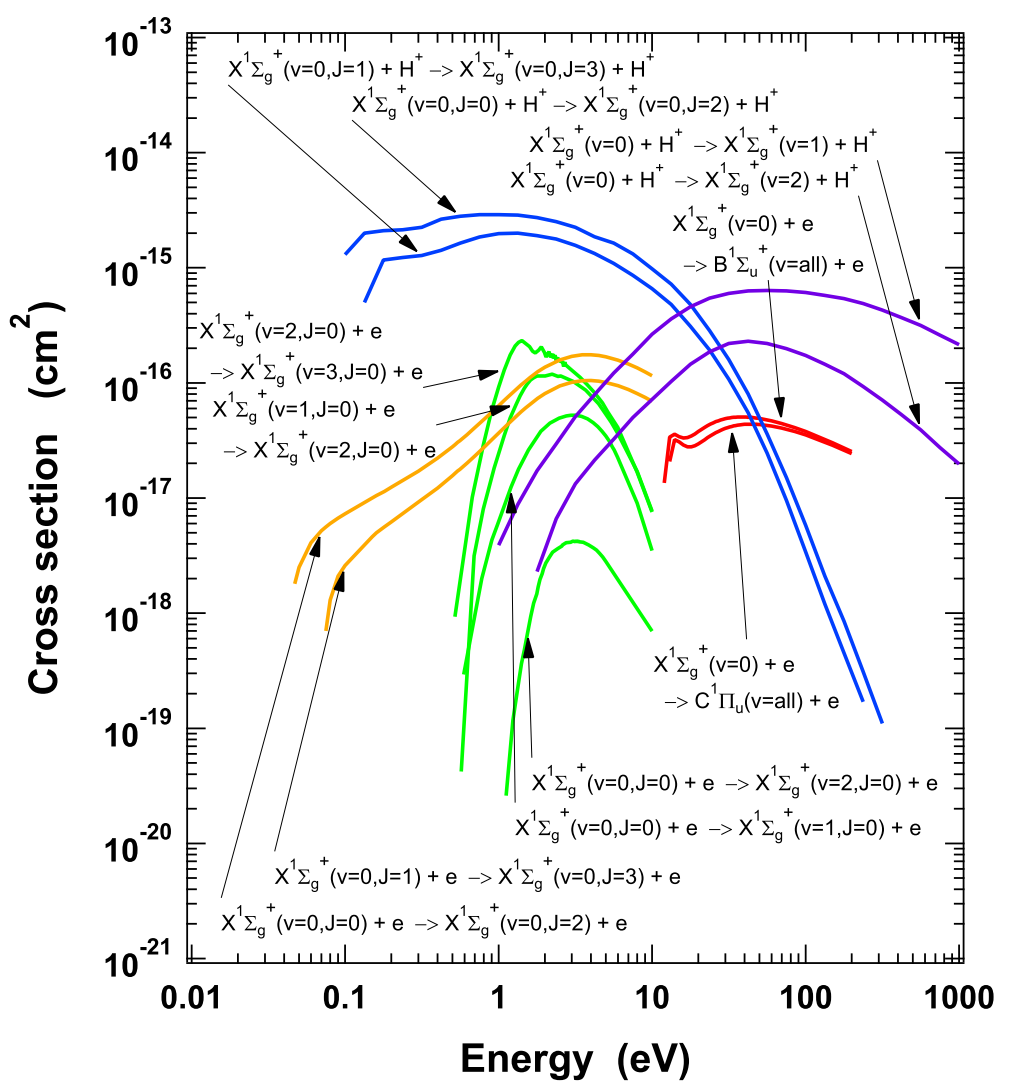

Figure 3. Some examples of the cross-sections for electron and proton impacts included in the present model. Data are taken from [49,53-55]. 


\subsubsection{Electron Impact Excitation between Electronic Excited States}

Table 8 shows available data on electron impact excitation between excited electronic states. As there are no available data for other transitions, we used cross-sections for the united atom helium (see Table 2) calculated using the convergent close-coupling (CCC) method [56]. The helium atom cross-sections corresponding to the transitions in Table 8 differ from those for the molecular transitions within a factor of $0.1-10$, this should be improved in the future.

Table 8. References for electron impact excitation cross-sections between electronic excited states.

\begin{tabular}{|c|c|c|c|}
\hline Transition & $\mathbf{e} \rightarrow \mathbf{e}^{\prime}$ & $\mathbf{e v} \rightarrow \mathbf{e}^{\prime}$ & $\mathbf{e v} \rightarrow \mathbf{e}^{\prime} \mathbf{v}^{\prime}$ \\
\hline$B^{1} \Sigma_{u}^{+} \rightarrow I^{1} \Pi_{g}$ & & [49] & \\
\hline $\begin{array}{l}a^{3} \Sigma_{g}^{+} \rightarrow c^{3} \Pi_{u} \\
a^{3} \Sigma_{g}^{+} \rightarrow d^{3} \Pi_{u} \\
c^{3} \Pi^{3} \rightarrow h^{3} \Sigma^{+}\end{array}$ & {$[57,58]$} & & {$[59,60]$} \\
\hline $\begin{array}{l}c^{3} \Pi_{u} \rightarrow b^{3} \Sigma_{u} \\
c^{3} \Pi_{u} \rightarrow g^{3} \Sigma_{g}^{+} \\
c^{3} \Pi_{u} \rightarrow h^{3} \Sigma_{g}^{+}\end{array}$ & {$[5 \%]$} & & $\begin{array}{l}{[59,60]} \\
{[59,60]}\end{array}$ \\
\hline
\end{tabular}

\subsubsection{Rovibrationally-Resolved Excitation}

The state-resolved electron impact rate coefficient from $(e, v, N)$ to $\left(e^{\prime}, v^{\prime}, N^{\prime}\right), R_{e v}^{e^{\prime} v^{\prime} N^{\prime}}$ subject to the Born-Oppenheimer approximation, is written as [17]:

$$
R_{e v N}^{e^{\prime} v^{\prime} N^{\prime}}=q_{e v N}^{e^{\prime} v^{\prime} N^{\prime}}\langle\sigma v\rangle_{e}^{e^{\prime}} a_{N}^{N^{\prime}} \delta_{a s}
$$

where $q_{e v e^{\prime} v^{\prime} N^{\prime}}$ is the Franck-Condon factor. And $\langle\sigma v\rangle_{e}^{e^{\prime}}$ is the rate coefficient for the transition between the electronic states. $\delta_{a s}$ is one or zero according to the selection rule from the symmetry of nuclear spin. The factor $a_{N}^{N^{\prime}}$ dominantly determines the rotational dependence. For the transition from the $X^{1} \Sigma_{g}^{+}$to the $d^{3} \Pi_{u}, a_{N}^{N^{\prime}}$ is given by [17] as:

$$
a_{N}^{N^{\prime}}=\sum_{r} \bar{Q}_{r}^{\prime}\left(2 N^{\prime}+1\right)\left(\begin{array}{ccc}
N^{\prime} & r & N \\
\Lambda^{\prime} & \Lambda-\Lambda^{\prime} & -\Lambda
\end{array}\right)^{2}
$$

where $\bar{Q}_{r}^{\prime}(r=1, \cdots, 4)$ have experimental values $0.76,0.122,0.1$ and 0.014 , respectively. The rate coefficient value is distributed to allow $J$ states according to their statistical weights. For other transitions, we distribute the rate coefficient into the final $J$ states within $\left|N-N^{\prime}\right|=0,1$ instead of $a_{N}^{N^{\prime}}$ in Equation (10). For transitions from the $X^{1} \Sigma_{g}^{+}$to the $B^{1} \Sigma_{u}^{+}$and $C^{1} \Pi_{u}$, we use the rate coefficients, which are vibrationally resolved for the upper and lower electronic states (see Table 6).

\subsection{Processes Involving $\mathrm{H}_{2}^{+}$}

Table 9 lists processes involving $\mathrm{H}_{2}^{+}$included in the present model. For ionization from the excited electronic states, we use the united atom helium data [56]. Figure 4 shows the cross-sections for the second process in Table 9.

Table 9. References for processes involving $\mathrm{H}_{2}^{+}$.

\begin{tabular}{ccc}
\hline Transition & $\mathbf{e v} \rightarrow \mathbf{e}^{\prime}$ & $\mathbf{e v} \rightarrow \mathbf{e}^{\prime} \mathbf{v}^{\prime}$ \\
\hline $\mathrm{H}_{2}\left(X^{1} \Sigma_{g}^{+}\right)+e \rightarrow \mathrm{H}_{2}^{+}\left(X^{2} \Sigma_{g}^{+}\right)+e$ & & {$[61]$} \\
$\mathrm{H}_{2}\left(X^{1} \Sigma_{g}^{+}\right)+\mathrm{H}^{+} \rightarrow \mathrm{H}_{2}^{+}\left(X^{2} \Sigma_{u}^{+}\right)+\mathrm{H}$ & {$[62,63]$} \\
\hline $\mathrm{H}_{2}\left(X^{1} \Sigma_{g}^{+}\right)+e \rightarrow \mathrm{H}_{2}^{+}\left(X^{2} \Sigma_{g}^{+}\right)+e \rightarrow \mathrm{H}(1 S)+\mathrm{H}^{+}+\mathrm{e}$ & {$[49]$} & \\
$\mathrm{H}_{2}\left(X^{1} \Sigma_{g}^{+}\right)+e \rightarrow \mathrm{H}_{2}^{+}\left(X^{2} \Sigma_{u}^{+}\right)+e \rightarrow \mathrm{H}(1 S)+\mathrm{H}^{+}+\mathrm{e}$ & {$[49]$} & \\
\hline
\end{tabular}




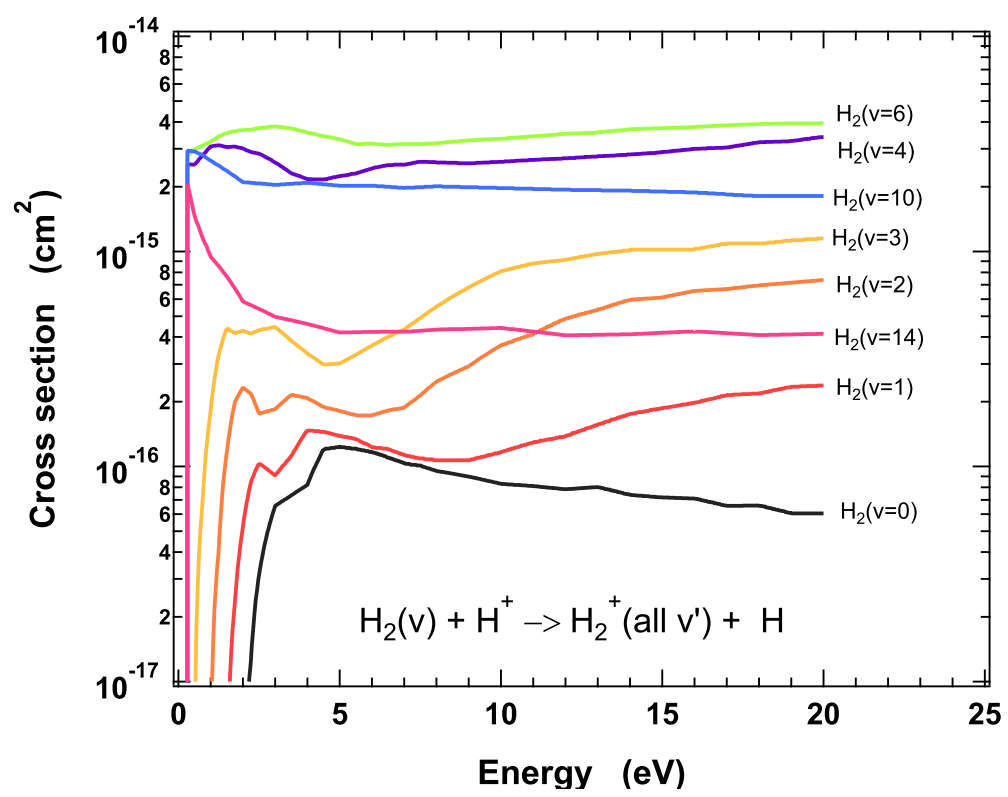

Figure 4. Cross-sections for $\mathrm{H}_{2}\left(X^{1} \Sigma_{g}^{+}\right)+\mathrm{H}^{+} \rightarrow \mathrm{H}_{2}^{+}\left(X^{2} \Sigma_{u}^{+}\right)+\mathrm{H}$. Data are taken from $[62,63]$.

\subsection{Pure Rotational Excitation in the $H_{2}\left(X^{1} \Sigma_{g}^{+}, v=0\right)$ by Electron Impact}

Cross-sections for the pure rotational excitation of the $(v=0, J=0) \rightarrow\left(v^{\prime}=0, J^{\prime}=2\right)$ and $(v=0, J=1) \rightarrow\left(v^{\prime}=0, J^{\prime}=3\right)$ transitions in [53] (see Figure 3) are included in the $\mathrm{H}_{2} \mathrm{CR}$ model. In [64], it is reported that the cross-section of the $(v=0, J=0) \rightarrow\left(v^{\prime}=0, J^{\prime}=4\right)$ transition is extremely small compared with that for $(v=0, J=0) \rightarrow\left(v^{\prime}=0, J^{\prime}=2\right)$ and that the cross-sections for $(v=0, J=1) \rightarrow\left(v^{\prime}=0, J^{\prime}=3\right),(v=0, J=2) \rightarrow\left(v^{\prime}=0, J^{\prime}=4\right)$ and $(v=0, J=3) \rightarrow\left(v^{\prime}=0, J^{\prime}=5\right)$ all lie within a factor of two. Thus, we include all other $\Delta J=2$ transition in $v=0$ assuming that the cross-sections are the same as that for $(v=0, J=1) \rightarrow$ $\left(v^{\prime}=0, J^{\prime}=3\right)$. For other vibrational states, we assume that rate coefficients for the pure rotational excitations are the same as those for $v=0$.

\subsection{Rovibrational Excitation by Electron Impact}

Cross-sections for the transition from $(v=0, J=0) \rightarrow\left(v^{\prime}=1 \sim 6, J=0\right)$ in the electronic ground state $X^{1} \Sigma_{g}^{+}$are calculated up to $6 \mathrm{eV}$ by considering $\mathrm{H}_{2}^{-}\left(X^{2} \Sigma_{u}^{+}\right)$resonance [54] (see Figure 3). Their rate coefficients are included in the present model.

In [54], the cross-sections for $(v=0, J=0) \rightarrow\left(v^{\prime}=1, J=0\right),(v=1, J=0) \rightarrow\left(v^{\prime}=2, J=0\right)$ and $(v=2, J=0) \rightarrow\left(v^{\prime}=3, J=0\right)$ in the $X^{1} \Sigma_{g}^{+}$state are also given. Their rate coefficients are included in the present model. Rate coefficients for other $(v, J=0) \rightarrow(v+1, J=0)(v \leq 6)$ transitions are obtained by assuming that their ratios as the same as their cross-sections at $4 \mathrm{eV}$ given in [54]. We assume that the rate coefficients for $(v, J=0) \rightarrow(v+1, J=0)(v>6)$ are the same as that for $(v=6, J=0) \rightarrow\left(v^{\prime}=7, J=0\right)$. We include the dissociation process from $\mathrm{H}_{2}\left(X^{1} \Sigma_{g}^{+}, v=14\right)$ to $\mathrm{H}_{2}\left(X^{1} \Sigma_{g}^{+}, v=\right.$ continuum $)$ assuming its rate coefficient is the same as that for $\mathrm{H}_{2}\left(X^{1} \Sigma_{g}^{+}, v=13\right) \rightarrow \mathrm{H}_{2}\left(X^{1} \Sigma_{g}^{+}, v=14\right)$. In the transitions in [54], the rotational state is fixed. We include $(v=0, J) \rightarrow\left(v^{\prime}=1 \sim 14, J^{\prime}=J, J \pm 2\right)$ and $(v, J) \rightarrow\left(v^{\prime}=v+1, J^{\prime}=J, J \pm 2\right)$ in the model assuming the cross-sections for $\Delta J=0, \pm 2$ transition are the same.

In [65], the electron impact vibrational excitation cross-sections $(v=0, J=0,4,8,12$, $16,20,23,26,29) \rightarrow\left(v^{\prime}=1, J^{\prime}=J\right)$ are given (see Figure 3$)$. We interpolate and extrapolate their rate coefficients to all $\mathrm{J}$. We include this process in addition to the above processes. 
Vibrational excitation cross-sections for all combinations of $(v) \rightarrow\left(v^{\prime}\right)$ are calculated in [66] by considering $\mathrm{H}_{2}^{-}\left(X^{2} \Sigma_{g}^{+}\right)$resonance. $\Delta J=0, \pm 2$ transition is included neglecting the rotational dependence of the cross-section.

\subsection{Vibrational and Rotational Excitation in the $\mathrm{H}_{2}\left(\mathrm{X}^{1} \Sigma_{g}^{+}, v, J\right)$ by Proton Impact}

For a pure rotational excitation, we include cross-sections for the $(v=0, J=0) \rightarrow(v=0, J=2)$ and $(v=0, J=1) \rightarrow(v=0, J=3)$ transitions from [55] (see Figure 3 ) in the $\mathrm{H}_{2} \mathrm{CR}$ model. Transitions for other $\Delta J=2$ in $v=0$ are also considered under the assumption that their cross-sections are the same as that for $(v,=0, J=1) \rightarrow(v=0, J=3)$. For other vibrational states, we assume that rate coefficients for the pure rotational excitations are the same as those for $v=0$.

Cross-sections for the vibrational transitions $(v=0) \rightarrow(v=1,2,3)$ are taken from [55] (see Figure 3). For other vibrational transitions, we include $(v) \rightarrow(v+1)$ by assuming that the rate coefficient ratios of $(v) \rightarrow(v+1)$ to $(v=0) \rightarrow(v=1)$ are the same as those for electron impact. We consider $\Delta J=0, \pm 2$ to be the final $J$ states. We neglect the rotational state dependence.

\subsection{Rotational Excitation in the $\mathrm{H}_{2}\left(\mathrm{X}^{1} \Sigma_{g}^{+}, v=0\right)$ by Molecular Hydrogen Impact}

As shown in Figure 5, a rate coefficient dataset for the rotational excitation in $\mathrm{H}_{2}\left(X^{1} \Sigma_{g}^{+}, v=0\right)$ arising from the collision of two molecules is given in [19]: the rate coefficients for collisions between a molecule with $J \leq 8$ and a molecule with $J=0$ or 1 are given for $2 \mathrm{~K} \leq T_{\mathrm{H}_{2}} \leq 10,000 \mathrm{~K}$. Data in [19] are included in the present model. We use the data from the $\mathrm{H}_{2}\left(X^{1} \Sigma_{g}^{+}, v=0\right)$ state for other vibrational states because no data are available.

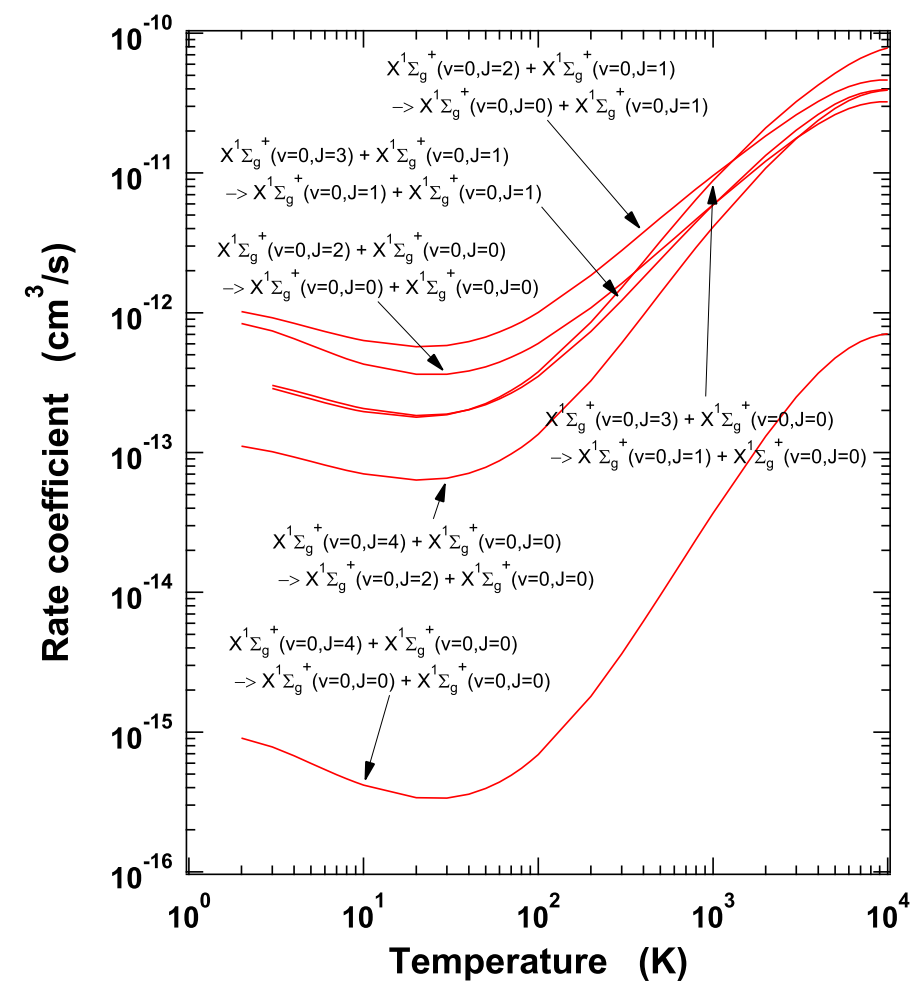

Figure 5. Rate coefficients for $\mathrm{H}_{2}\left(X^{1} \Sigma_{g}^{+}, v=0, J\right)+\mathrm{H}_{2}\left(X^{1} \Sigma_{g}^{+}, v=0, J=0,1\right) \rightarrow \mathrm{H}_{2}\left(X^{1} \Sigma_{g}^{+}, v=0, J^{\prime}\right)$ $+\mathrm{H}_{2}\left(X^{1} \Sigma_{g}^{+}, v=0, J=0,1\right)$. Data are taken from [19]. 
3.10. Rovibrational Excitation in the $\mathrm{H}_{2}\left(X^{1} \Sigma_{g}^{+}, v, J\right)$ by Atomic Hydrogen Impact

A cross-sectional dataset for the rovibrational excitation in $X^{1} \Sigma_{g}^{+}$owing to the collision of atomic hydrogen is given in [67] (see Figure 6). Rate coefficients in the present model are calculated using this dataset for transitions among $v=0-6, J=0-23$ in the barycentric collision energy range 200-60,000 K.

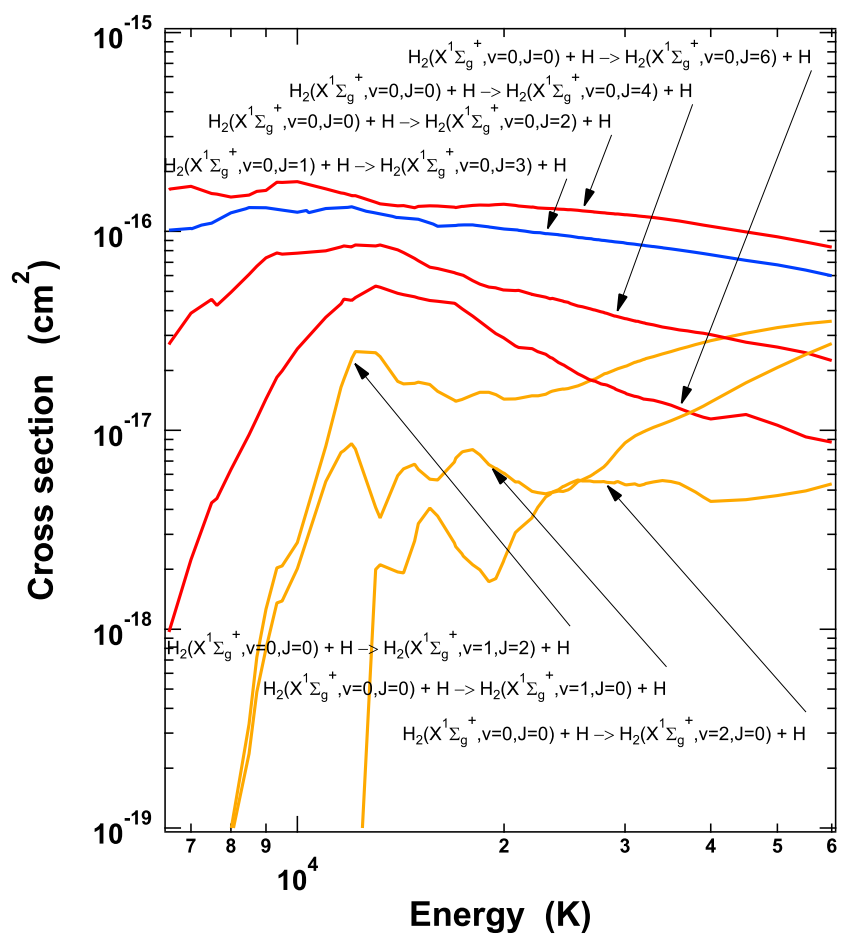

Figure 6. Cross-sections for $\mathrm{H}_{2}\left(X^{1} \Sigma_{g}^{+}, v, J\right)+\mathrm{H} \rightarrow \mathrm{H}_{2}\left(X^{1} \Sigma_{g}^{+}, v^{\prime}, J^{\prime}\right)+\mathrm{H}$. Data are taken from [67].

\subsection{Dissociation of $\mathrm{H}_{2}\left(X^{1} \Sigma_{g}^{+}, v\right)$ by Atomic Hydrogen Impact}

Vibrationally-resolved rate coefficients for the dissociation of $\mathrm{H}_{2}\left(X^{1} \Sigma_{g}^{+}, v\right)$ by atomic hydrogen impact given in [68] (Figure 7) are included in the present model. Here, rotational dependence is neglected because no relevant data are available.

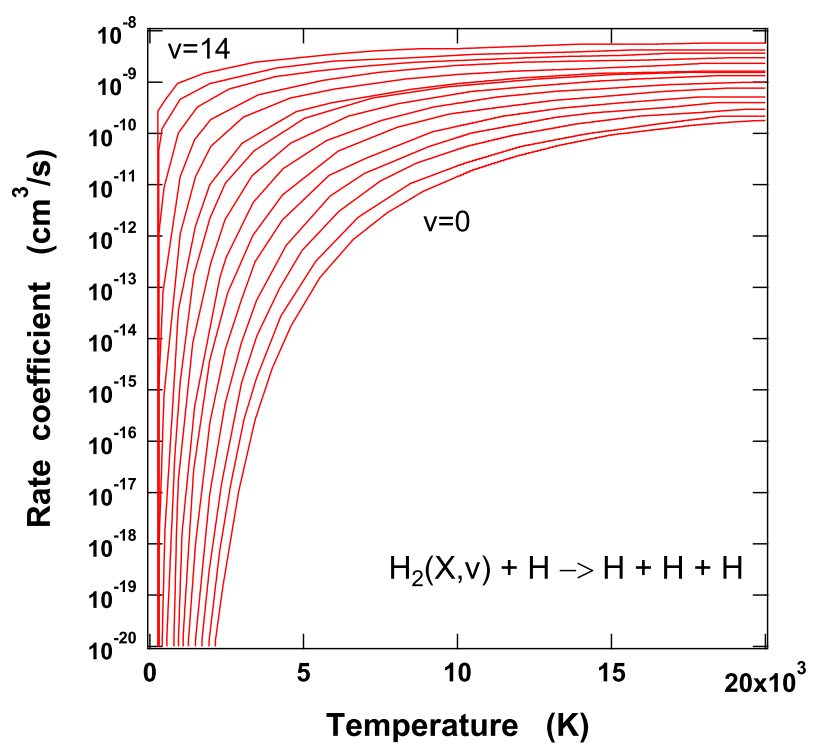

Figure 7. Rate coefficients for $\mathrm{H}_{2}\left(X^{1} \Sigma_{g}^{+}, v\right)+\mathrm{H} \rightarrow \mathrm{H}+\mathrm{H}+\mathrm{H}$. Data are taken from [68]. 


\subsection{Dissociative Attachment by Electron Impact}

As shown in Figure 8, the dissociative attachment rate coefficient is calculated in [69] by considering $\mathrm{H}_{2}^{-}\left(X^{2} \Sigma_{u}^{+}\right)$resonance. The complete dataset from all rovibrational states in the $\mathrm{H}_{2}\left(X^{1} \Sigma_{g}^{+}, v, J\right)$ is included in the CR model. The dissociative attachment cross-section from all vibrational states in $\mathrm{H}_{2}\left(X^{1} \Sigma_{g}^{+}, v\right)$ calculated in [70] by considering $\mathrm{H}_{2}^{-}\left(X^{2} \Sigma_{g}^{+}\right)$resonance is also included in the CR model. In this case, the rotational dependence is neglected because of a lack of data.

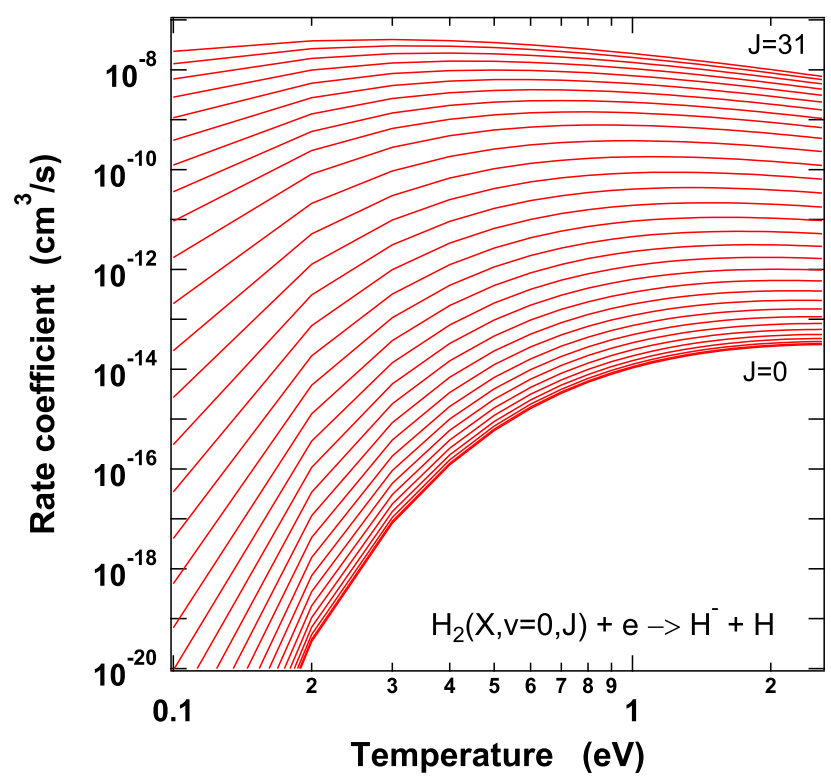

Figure 8. Rate coefficients for $\mathrm{H}_{2}\left(X^{1} \Sigma_{g}^{+}, v, J\right)+e \rightarrow \mathrm{H}_{2}^{-}\left(X^{2} \Sigma_{u}^{+}\right)+e \rightarrow \mathrm{H}(1)+\mathrm{H}^{-}$. Data are taken from [69].

\subsection{Dissociative Processes Starting from the $\mathrm{H}_{2}\left(X^{1} \Sigma_{g}^{+}, v\right)$ by Electron Impact}

The dissociative processes in Table 10 are included in the model in order to determine the population of the $\mathrm{H}_{2}\left(X^{1} \Sigma_{g}^{+}, v, J\right)$; once again, rotational dependence is neglected.

Table 10. References for the electron impact dissociative processes starting from $\mathrm{H}_{2}\left(X^{1} \Sigma_{g}^{+}\right)$.

\begin{tabular}{cc}
\hline Transition & $\mathrm{ev} \rightarrow$ \\
\hline $\mathrm{H}_{2}\left(X^{1} \Sigma_{g}^{+}\right)+\mathrm{e} \rightarrow \mathrm{H}_{2}\left(B^{1} \Sigma_{u}^{+}\right)+\mathrm{e} \rightarrow \mathrm{H}(1)+\mathrm{H}(2)+\mathrm{e}$ & {$[49]$} \\
$\mathrm{H}_{2}\left(X^{1} \Sigma_{g}^{+}\right)+\mathrm{e} \rightarrow \mathrm{H}_{2}\left(B^{\prime 1} \Sigma_{u}^{+}\right)+\mathrm{e} \rightarrow \mathrm{H}(1)+\mathrm{H}(2)+\mathrm{e}$ & {$[49]$} \\
$\mathrm{H}_{2}\left(X^{1} \Sigma_{g}^{+}\right)+\mathrm{e} \rightarrow \mathrm{H}_{2}\left(B^{\prime \prime 1} \Sigma_{u}^{+}\right)+\mathrm{e} \rightarrow \mathrm{H}(1)+\mathrm{H}(3)+\mathrm{e}$ & {$[49]$} \\
\hline $\mathrm{H}_{2}\left(X^{1} \Sigma_{g}^{+}\right)+\mathrm{e} \rightarrow \mathrm{H}_{2}\left(C^{1} \Pi_{u}\right)+\mathrm{e} \rightarrow \mathrm{H}(1)+\mathrm{H}(2)+\mathrm{e}$ & {$[49]$} \\
$\mathrm{H}_{2}\left(X^{1} \Sigma_{g}^{+}\right)+\mathrm{e} \rightarrow \mathrm{H}_{2}\left(D^{1} \Pi_{u}\right)+\mathrm{e} \rightarrow \mathrm{H}(1)+\mathrm{H}(3)+\mathrm{e}$ & {$[49]$} \\
$\mathrm{H}_{2}\left(X^{1} \Sigma_{g}^{+}\right)+\mathrm{e} \rightarrow \mathrm{H}_{2}\left(D^{\prime 1} \Pi_{u}\right)+\mathrm{e} \rightarrow \mathrm{H}(1)+\mathrm{H}(4)+\mathrm{e}$ & {$[49]$} \\
\hline
\end{tabular}

\section{Results and Discussion}

We applied the $\mathrm{H}_{2} \mathrm{CR}$ model to a fusion detached plasma as an example of the application of the present model using the following parameters taken from a SlimCS DEMO detached plasma simulation [12,13]: $T_{\mathrm{e}}=T_{\mathrm{H}^{+}}=T_{\mathrm{H}}=2 \mathrm{eV}, n_{\mathrm{e}}=n_{\mathrm{H}^{+}}=10^{16} \mathrm{~cm}^{-3}$ and $n_{\mathrm{H}}=10^{15} \mathrm{~cm}^{-3}$. In the present calculation, background molecular hydrogen, which contributes to the rotational excitation, is limited to $\mathrm{H}_{2}\left(X^{1} \Sigma_{g}^{+}, v=0, J=0,1\right)$, and the kinetic temperature and total density are fixed at $\mathrm{T}_{\mathrm{H}_{2}}=1 \mathrm{eV}$ and $n_{\mathrm{H}_{2}}=10^{15} \mathrm{~cm}^{-3}$, respectively. 
The present code solves the rovibrational populations of the $X^{1} \Sigma_{g}^{+}, a^{3} \Sigma_{g}^{+}$and $c^{3} \Pi_{u}^{-}$time dependently. The values of $\tau(p)$ from Equation (6) for the rovibrational states of $X^{1} \Sigma_{g}^{+}$are $1 \times 10^{-9} \sim 1 \times 10^{-8} \mathrm{~s}$. The values of $\tau_{\max }$ in the electronic excited states is $2 \times 10^{-9} \mathrm{~s}$ for a level in the $a^{3} \Sigma_{g}^{+}$. Except for the $a^{3} \Sigma_{g}^{+}, \tau$ is smaller than $1 \times 10^{-10} \mathrm{~s}$. In this case, the $c^{3} \Pi_{u}^{-}(v=0)$, known as the metastable state, does not have a significantly larger $\tau(p)$ than the other levels owing to the large value of $n_{\mathrm{e}}$. The Runge-Kutta method was used with the time step of $\Delta t=10^{-11} \mathrm{~s}$. The QSS was applied to the rovibrational states in the other electronic excited states.

Figure 9 shows the population of the rotational states in $\mathrm{H}_{2}\left(X^{1} \Sigma_{g}^{+}, v=0\right)$ as a function of time. The initial population of $300 \mathrm{~K}$ Boltzmann distribution is given at $t=0 \mathrm{~s}$. Initial total $\mathrm{H}_{2}$ density was set at $1 \mathrm{~cm}^{-3}$. In the present case, the distribution may be understood to be that of a gas puffed molecule. In modeling the recycling of a molecule at a wall, a proper rovibrational distribution should be given.

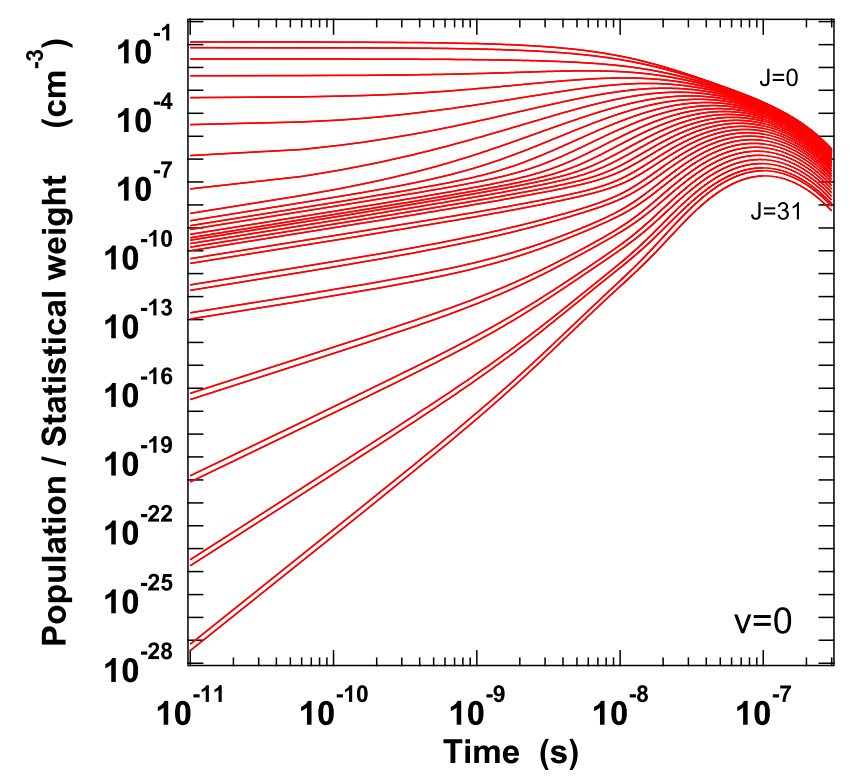

Figure 9. Population divided by the statistical weight of the rotational states in $X^{1} \Sigma_{g}^{+}(v=0)$.

Figure 10a-c shows the rovibrational population distributions at $t=10^{-10} \mathrm{~s}, t=10^{-8} \mathrm{~s}$ and $t=10^{-7} \mathrm{~s}$, respectively. Figure $10 \mathrm{~b}, \mathrm{c}$ suggests a different populating mechanism from that in Figure 10a.

Figure 11a-c shows the inflow to each rovibrational level at $t=10^{-10} \mathrm{~s}, t=10^{-8} \mathrm{~s}$ and $t=10^{-7} \mathrm{~s}$, respectively. At $t=10^{-10} \mathrm{~s}$, the dominant inflow to each level of $0 \leq J \leq 8$ is the electron impact transition in $\mathrm{H}_{2}\left(X^{1} \Sigma_{g}^{+}, v, J\right)$. The origin of the shoulder-like profile for $J>8$ in Figure 11a is the atomic hydrogen impact transition from lower lying $J=0,1$ states with the large population. The $J$ dependence of the contributions of molecular and atomic collisions can be attributed to the differences in the $J$ dependence of the rate coefficients or cross-sections in Figures 5 and 6 . The transition with photoemission to the $\mathrm{H}_{2}\left(X^{1} \Sigma_{g}^{+}, v, J\right)$ is limited to $J=14$ because the upper limit of $J^{\prime}$ in the electronic excited states is $J^{\prime}=13$ in the $B^{1} \Sigma_{u}^{+}\left(v^{\prime}=2\right)$ state. As shown in Figure $11 b, c$, increasing the excited rovibrational state population leads to an increase in electron impact transition among these states.

Figure 12a-c shows the outflow from each rovibrational level at $t=10^{-10} \mathrm{~s}, t=10^{-8} \mathrm{~s}$ and $t=10^{-7} \mathrm{~s}$, respectively. As the highly excited rovibrational state population increases, the dissociative attachment increases. Finally, as shown in Figures 11c and 12c, the population distribution is dominated by the large flow in the $X^{1} \Sigma_{g}^{+}$from electron impact and quenching processes including the dissociative attachment. After $t=10^{-7} \mathrm{~s}$, the total population decreases with the relative rovibrational population in $X^{1} \Sigma_{g}^{+}$kept nearly constant. 

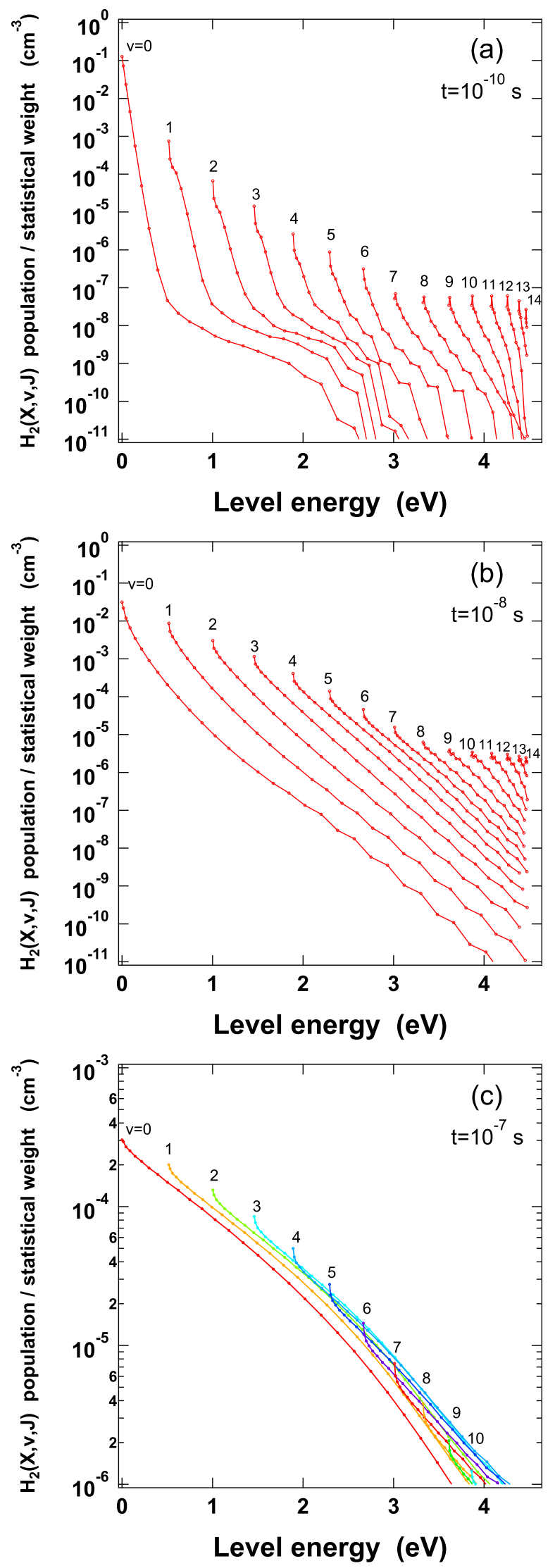

Figure 10. Population of the $X^{1} \Sigma_{g}^{+}(v, J)$ divided by the statistical weight: $(\mathbf{a}) t=10^{-10} \mathrm{~s}$; (b) $t=10^{-8} \mathrm{~s}$; and (c) $t=10^{-7} \mathrm{~s}$. 

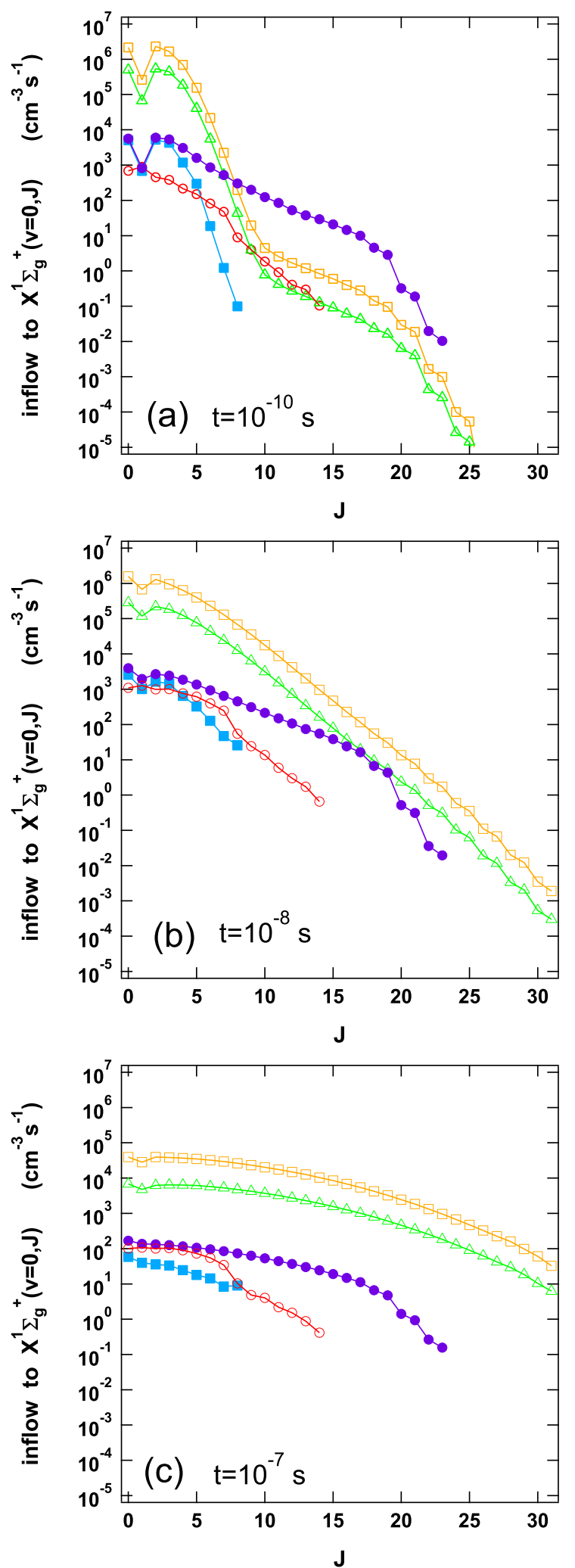

Figure 11. Inflow to each rovibrational level divided by statistical weight. The total inflow from all initial states is shown. (a) $t=10^{-10} \mathrm{~s}$; (b) $t=10^{-8} \mathrm{~s}$; and (c) $t=10^{-7} \mathrm{~s}$. (Open circle) Emission from the electronic excited states, (open square) electron collision transition in $X^{1} \Sigma_{g}^{+}$, (open triangle) proton collision transition in $X^{1} \Sigma_{g}^{+}$, (closed circle) atomic collision transition in $X^{1} \Sigma_{g}^{+}$, (closed square) molecular collision transition in $X^{1} \Sigma_{g}^{+}$. 

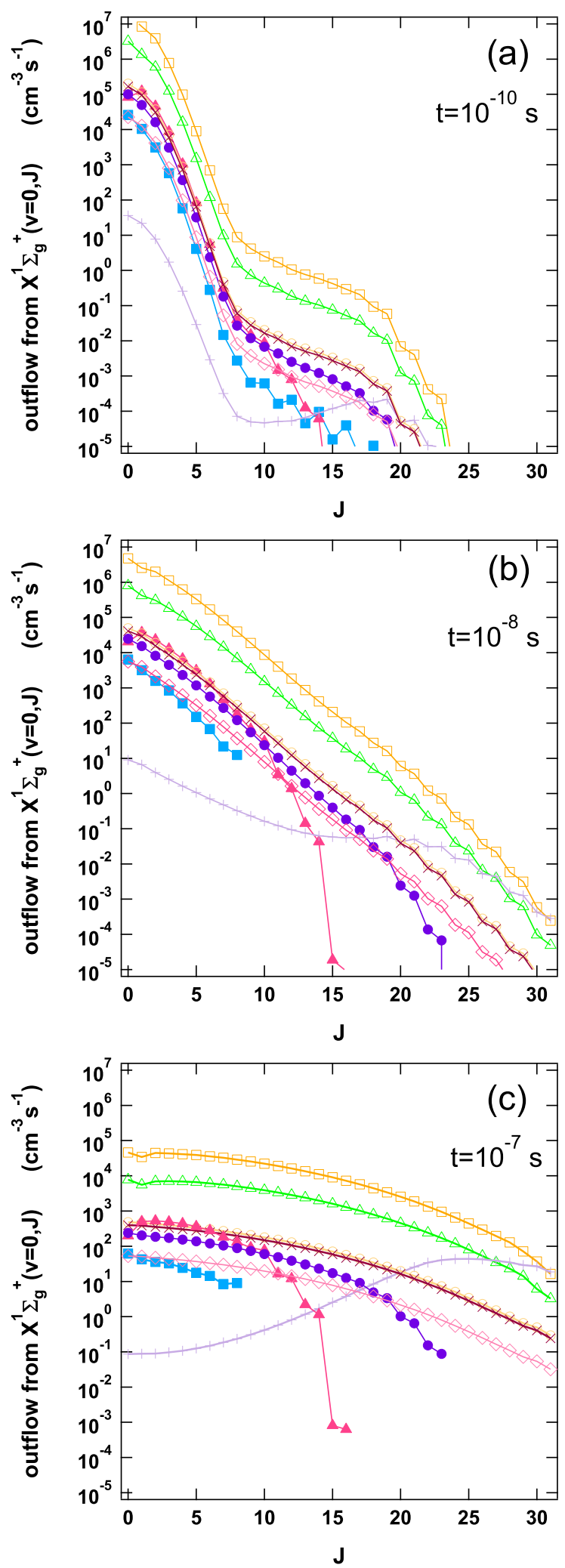

Figure 12. Outflow from each rovibrational level divided by statistical weight. The total outflow to all final states is shown. (a) $t=10^{-10} \mathrm{~s}$; (b) $t=10^{-8} \mathrm{~s}$; and (c) $t=10^{-7} \mathrm{~s}$. (Open square) Electron collision transition in $X^{1} \Sigma_{g}^{+}$, (open triangle) proton collision transition in $X^{1} \Sigma_{g}^{+}$, (closed square) molecular collision transition in $X^{1} \Sigma_{g}^{+}$, (closed circle) atomic collision transition in $X^{1} \Sigma_{g}^{+}$, (closed triangle) electron collision to the excited electronic states, (plus) dissociative attachment, (open circle) dissociation through the excited electronic states including $b^{3} \Sigma_{u}^{+}$, (cross) charge transfer to $\mathrm{H}^{+}$, (open diamond) dissociation by atomic hydrogen impact. 
Figure 13 shows the vibrational population distributions obtained by summing all rotational populations. The distribution is not expressed as a simple Boltzmann distribution.

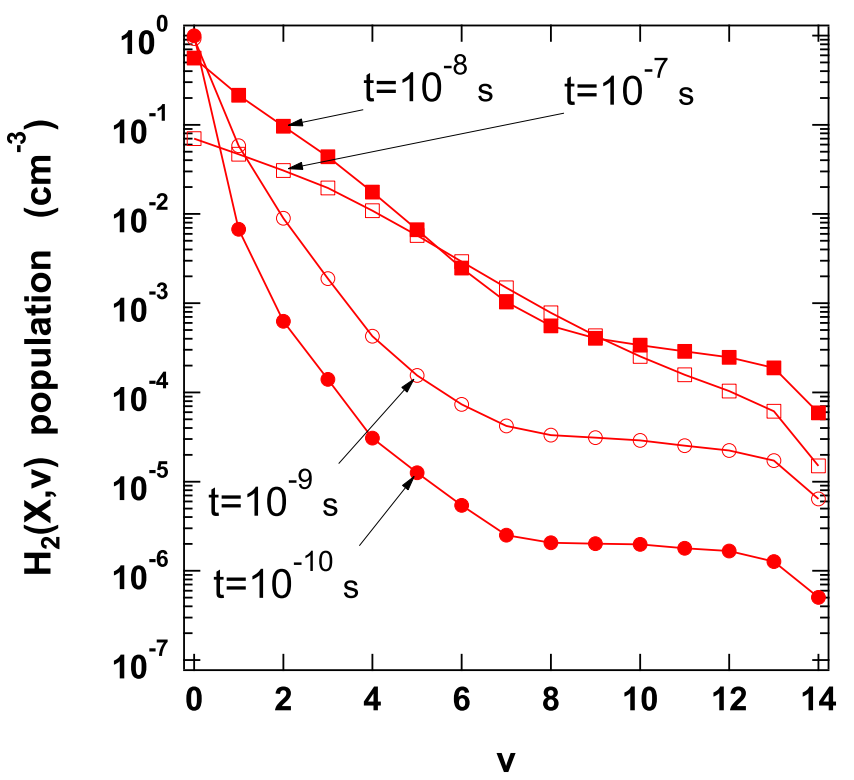

Figure 13. Vibrational population distribution. Rotational populations in each vibrational state are summed.

Figure 14 shows the effective rate coefficients for MAR (P1-P3) and (P4-P7) in Table 11 and other processes in which atomic hydrogen is produced (P8-P12) and (P14-P15). Here, the effective rate coefficients are defined by:

$$
\begin{aligned}
\frac{d n_{\mathrm{H}}}{d t} & =P_{\mathrm{H}_{2}}^{\mathrm{H}} n_{\mathrm{H}_{2}} n_{\mathrm{e}} \\
\frac{d n_{\mathrm{H}}}{d t} & =P_{\mathrm{H}^{+}}^{\mathrm{H}} n_{\mathrm{H}^{+}} n_{\mathrm{e}}
\end{aligned}
$$

where $P_{\mathrm{H}_{2}}^{\mathrm{H}}(\mathrm{P} 1-\mathrm{P} 12)$ and $P_{\mathrm{H}^{+}}^{\mathrm{H}}(\mathrm{P} 14-\mathrm{P} 15)$ are the effective rate coefficients for the production of atomic hydrogen from $\mathrm{H}_{2}$ and $\mathrm{H}^{+}$, respectively, including the contributions of their excited states, $n_{\mathrm{H}}$ is the population density of the ground state atom, $n(1)$ in Equation (4), $n_{\mathrm{H}_{2}}$ is total population density of $\mathrm{H}_{2}$ including the ground and excited states, and $P_{\mathrm{H}^{+}}^{\mathrm{H}}$ is the same as $\alpha_{\mathrm{CR}}$ in Equation (5).

The effective rate coefficients are calculated using cross-section data and models in the references in Table 11, in addition to the present $\mathrm{H}_{2}$ CR model. In (P3, P7, P12, P15), the atomic hydrogen CR model $[14,15]$ is used to calculate population flows into the ground state from excited states. The vibrationally-resolved CR model for $\mathrm{H}_{2}^{+}$[7] that includes processes (P4-P6) and (P16) in Table 11 is used to calculate the population distribution of $\mathrm{H}_{2}{ }^{+}\left(X^{2} \Sigma_{g}^{+}, v\right)$ assuming the QSS. Rotational levels are not considered in the $\mathrm{H}_{2}{ }^{+}$CR model. For processes (P11) and (P12), we use dissociative excitation data derived from experimental emission cross-sections given in [2,71] instead of those in the references in Table 10 in order to include the contribution from all of the excited states of the $\mathrm{H}_{2}$ molecule. The rovibrational dependence is not included in the present calculation owing to a lack of relevant information.

Figure 14 shows that the effective rate coefficients for (P1-P3) and (P4-P7) increase with the excited rovibrational population in $X^{1} \Sigma_{g}^{+}$. For $t>10^{-7} \mathrm{~s}$, the sum of the effective rate coefficients of the two types of MAR is larger by a factor of approximately 25 than that at $t=10^{-11} \mathrm{~s}$. Furthermore, MAR cannot be neglected relative to the well-known dissociative process (P8) as a source of atomic hydrogen after time $t>10^{-8} \mathrm{~s}$.

In this study, we used the rate coefficient of dissociative attachment based on the nonlocal resonance model [69]. According to this model, it is pointed out that non-resonant scattering enhances 
the rate coefficient [72] and, although a complete dataset including it is not available, this effect may enhance the effective rate coefficient of (P1-P3) by an approximate factor of two beyond the value calculated here.

The photoemission transition from triplet electronic excited states (P10) (see Table 5) is also important for atom production. The populations of the upper states of the photoemission transitions in (P9) and (P10) increase up to $t \sim 10^{-8} \mathrm{~s}$. In a later time, these populations decrease because the relative population of lower rotational states in $X^{1} \Sigma_{g}^{+}$, which are the sources of the populations of the upper states of the photoemission, decreases; the electron impact excitation for $\Delta J=0, \pm 2$ is considered and the number of the rotational states of the upper state is smaller than that of the $X^{1} \Sigma_{g}^{+}$.

In this calculation, as the quenching from higher lying rotational states, only the dissociative attachment is effectively considered, because the rotational dependence of the cross-section is not included for other quenching processes. Furthermore, the electron impact dissociation through the $X^{1} \Sigma_{g}^{+}$continuum, which may play an important role, is not included here because no relevant data are available. If the dissociative attachment process is not dominant, the population density becomes smaller, which in turn reduces the effective rate coefficient for the MAR starting from the attachment process. The quenching cross-section from the higher rotational states should be studied in detail in future research. For precise calculation of the effective rates, precise cross-section data for the various transitions in $X^{1} \Sigma_{g}^{+}$will also be indispensable.

Table 11. Molecular-assisted recombination (P1-P3 and P4-P7) and some other processes. $p$ is the principal quantum number of the $\mathrm{H}$ atom. $\mathrm{H}^{*}$ and $\mathrm{H}_{2}^{*}$ denote the excited atom and molecule, respectively.

\begin{tabular}{|c|c|c|}
\hline Label & Process & Reference \\
\hline $\begin{array}{l}\text { (P1) } \\
\text { (P2) } \\
\text { (P3) }\end{array}$ & $\begin{array}{c}\mathrm{H}_{2}\left(X^{1} \Sigma_{g}^{+}, v, J\right)+\mathrm{e} \rightarrow \mathrm{H}_{2}^{-} \rightarrow \mathrm{H}(p=1 \text { or } 2)+\mathrm{H}^{-} \\
\mathrm{H}^{-}+\mathrm{H}^{+} \rightarrow \mathrm{H}(1)+\mathrm{H}(p=2 \text { or } 3) \\
\mathrm{H}(p=2 \text { or } 3) \rightarrow \mathrm{H}^{*} \rightarrow \mathrm{H}(1)\end{array}$ & $\begin{array}{l}{[69,70]} \\
{[73]} \\
{[14,15]}\end{array}$ \\
\hline $\begin{array}{l}(\mathrm{P} 4) \\
(\mathrm{P} 5) \\
(\mathrm{P} 6) \\
(\mathrm{P} 7)\end{array}$ & $\begin{array}{c}\mathrm{H}_{2}\left(X^{1} \Sigma_{g}^{+}, v\right)+\mathrm{H}^{+} \rightarrow \mathrm{H}_{2}^{+}\left(X^{2} \Sigma_{g}^{+}, v^{\prime}\right) \rightarrow \mathrm{H}(1) \\
\mathrm{H}_{2}{ }^{+}\left(X^{2} \Sigma_{g}^{+}, v^{\prime}\right)+\mathrm{e} \rightarrow \mathrm{H}_{2}^{+}\left(X^{2} \Sigma_{g}^{+}, v^{\prime \prime}\right)+\mathrm{e} \\
\mathrm{H}_{2}^{+}\left(X^{2} \Sigma_{g}^{+}, v^{\prime \prime}\right)+\mathrm{e} \rightarrow \mathrm{H}(1)+\mathrm{H}(p \geq 2) \\
\mathrm{H}(p \geq 2) \rightarrow \mathrm{H}^{*} \rightarrow \mathrm{H}(1)\end{array}$ & $\begin{array}{c}{[62,63]} \\
{[74]} \\
{[74]} \\
{[14,15]}\end{array}$ \\
\hline (P8) & $\mathrm{H}_{2}\left(X^{1} \Sigma_{g}^{+}, v\right)+\mathrm{e} \rightarrow \mathrm{H}_{2}\left(b^{3} \Sigma_{u}^{+}\right)+\mathrm{e} \rightarrow \mathrm{H}(1)+\mathrm{H}(1)$ & [49] \\
\hline (P9) & $\mathrm{H}_{2}{ }^{*}($ singlet $, v, J) \rightarrow \mathrm{H}_{2}\left(X^{1} \Sigma_{g}^{+}\right)+h v \rightarrow \mathrm{H}(1)+\mathrm{H}(1)$ & - \\
\hline (P10) & $\mathrm{H}_{2}{ }^{*}($ triplet $, v, J) \rightarrow \mathrm{H}_{2}\left(b^{3} \Sigma_{u}^{+}\right)+h v \rightarrow \mathrm{H}(1)+\mathrm{H}(1)$ & - \\
\hline $\begin{array}{l}\text { (P11) } \\
\text { (P12) }\end{array}$ & $\begin{array}{c}\mathrm{H}_{2}\left(X^{1} \Sigma_{g}^{+}\right)+\mathrm{e} \rightarrow \mathrm{H}(1)+\mathrm{H}(p \geq 2) \\
\mathrm{H}(p \geq 2) \rightarrow \mathrm{H}^{*} \rightarrow \mathrm{H}(1)\end{array}$ & $\begin{array}{l}{[2,71]} \\
{[2,71]}\end{array}$ \\
\hline (P13) & $\mathrm{H}_{2}\left(X^{1} \Sigma_{g}^{+}, v\right)+\mathrm{H} \rightarrow \mathrm{H}+\mathrm{H}+\mathrm{H}$ & [68] \\
\hline $\begin{array}{l}(\mathrm{P} 14) \\
(\mathrm{P} 15)\end{array}$ & $\begin{array}{c}\mathrm{H}^{+}+\mathrm{e} \rightarrow \mathrm{H}(1) \\
\mathrm{H}^{+}+\mathrm{e} \rightarrow \mathrm{H}^{*} \rightarrow \mathrm{H}(1)\end{array}$ & $\begin{array}{l}{[14,15]} \\
{[14,15]}\end{array}$ \\
\hline (P16) & $\mathrm{H}_{2}^{+}\left(X^{2} \Sigma_{g}^{+}, v^{\prime}\right)+\mathrm{e} \rightarrow \mathrm{H}(1)+\mathrm{H}^{+}+\mathrm{e}$ & {$[74]$} \\
\hline $\begin{array}{l}\text { (P17) } \\
\text { (P18) }\end{array}$ & $\begin{array}{c}\mathrm{H}_{2}^{+}+\mathrm{H}_{2} \rightarrow \mathrm{H}_{3}^{+}+\mathrm{H} \\
\mathrm{H}_{3}{ }^{+}+\mathrm{e} \rightarrow \mathrm{H}_{2}\left(X^{1} \Sigma_{g}^{+}, v, J\right)+\mathrm{H} \quad \text { or } \quad \mathrm{H}+\mathrm{H}+\mathrm{H}\end{array}$ & {$[75]$} \\
\hline $\begin{array}{l}(\mathrm{P} 19) \\
(\mathrm{P} 20)\end{array}$ & $\begin{array}{c}\mathrm{H}_{2}^{+}+e \rightarrow \mathrm{H}_{2}\left(X^{1} \Sigma_{g}^{+}, v, J\right) \\
\mathrm{H}_{2}^{+}+e \rightarrow \mathrm{H}_{2}{ }^{*} \rightarrow \mathrm{H}_{2}\left(X^{1} \Sigma_{g}^{+}, v, J\right)\end{array}$ & \\
\hline$(\mathrm{P} 21)$ & $\mathrm{H}+\mathrm{H}+\mathrm{H} \rightarrow \mathrm{H}_{2}\left(X^{1} \Sigma_{g}^{+}, v, J\right)+\mathrm{H}$ & [77] \\
\hline (P22) & $\mathrm{H}+\mathrm{H}_{\mathrm{wall}} \rightarrow \mathrm{H}_{2}\left(X^{1} \Sigma_{g}^{+}, v, J\right)$ & [77] \\
\hline
\end{tabular}




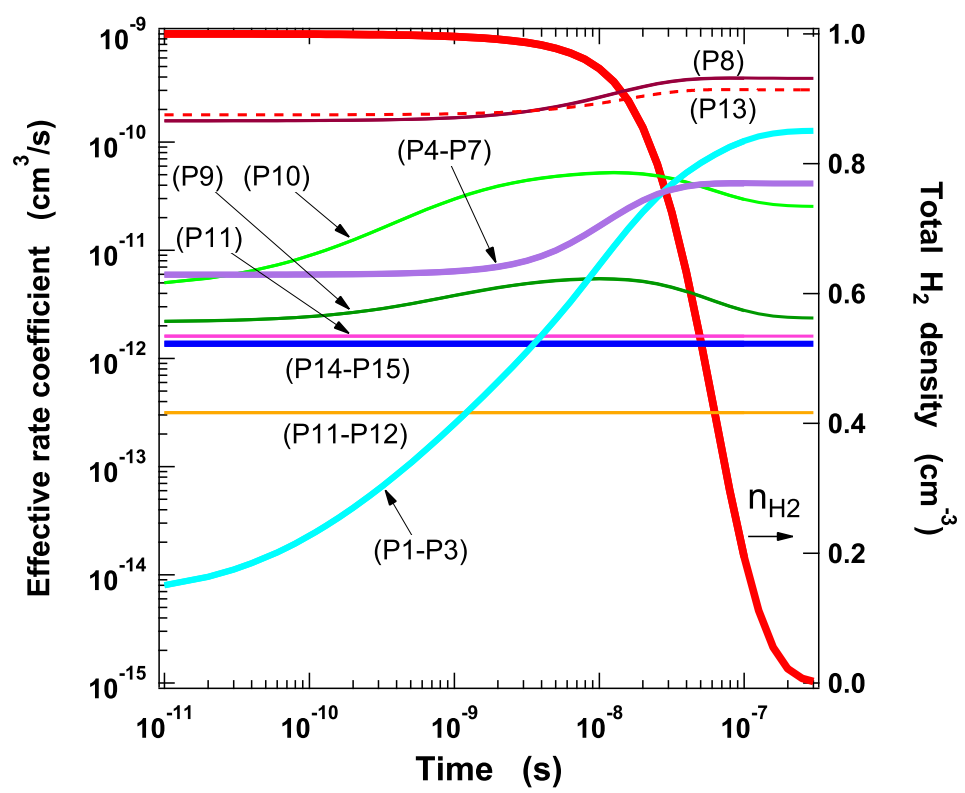

Figure 14. Details of $P_{\mathrm{H}_{2}}^{\mathrm{H}}$ in Equation (12). Effective rate coefficients for molecular-assisted recombination (MAR) and other processes for the production of atomic hydrogen (P1-P12) and (P14-P15) in Table 11 are shown. The $P_{\mathrm{H}_{2}}^{\mathrm{H}}$ for (P13), defined as $\frac{d n_{\mathrm{H}}}{d t}=P_{\mathrm{H}_{2}}^{\mathrm{H}} n_{\mathrm{H}_{2}} n_{\mathrm{H}}$, is also shown. The values for (P8), (P9), (P10) and (P13) in this figure should be multiplied by two because two atoms are produced.

Figure 15a-c shows an example of the emission spectra of molecular hydrogen calculated from the obtained rovibrational population distribution of the excited electronic states by multiplying the spontaneous transition coefficient and photon energy. At present, the uncertainty in the electron impact excitation cross-section between the electronic states is large. For qualitative spectroscopic diagnostic, a more reliable dataset is necessary.

Here, we would like to discuss the application of the model presented in this paper to more general cases. In this study, we addressed processes starting from the $\mathrm{H}_{2}\left(X^{1} \Sigma_{g}^{+}, v, J\right)$. In future work, a model for the recombining component starting from $\mathrm{H}_{2}{ }^{+}$, (P19-P20) in Table 11, should be constructed for lower electron temperature plasmas. Furthermore, recombination starting from atomic hydrogen, (P21) in Table 11, may contribute to the rovibrational population of the $\mathrm{H}_{2}\left(X^{1} \Sigma_{g}^{+}, v, J\right)$ in high atomic density plasmas [77]. In considering the recycling of molecules at a wall (P22), a proper initial population distribution at the wall should be given to the model. As for the present calculation, interpreting it as that for the history of a gas puffed molecule, the result is valid regardless of the negligence of the above processes. Figure 16 shows the rate coefficients of (P6) + (P16) and (P17) in Table 11. When the electron density is much smaller than the molecular density, $\mathrm{H}_{3}^{+}$may be produced by (P17), which is a competing process of $(\mathrm{P} 6)+(\mathrm{P} 16)$. In this case, the dissociative process from $\mathrm{H}_{3}^{+}$ (P18) may contribute to the population of $\mathrm{H}_{2}\left(\mathrm{X}^{1} \Sigma_{g}^{+}, v, J\right)$. Production of the rovibrationally-resolved cross-section for this process is indispensable for such plasmas. 

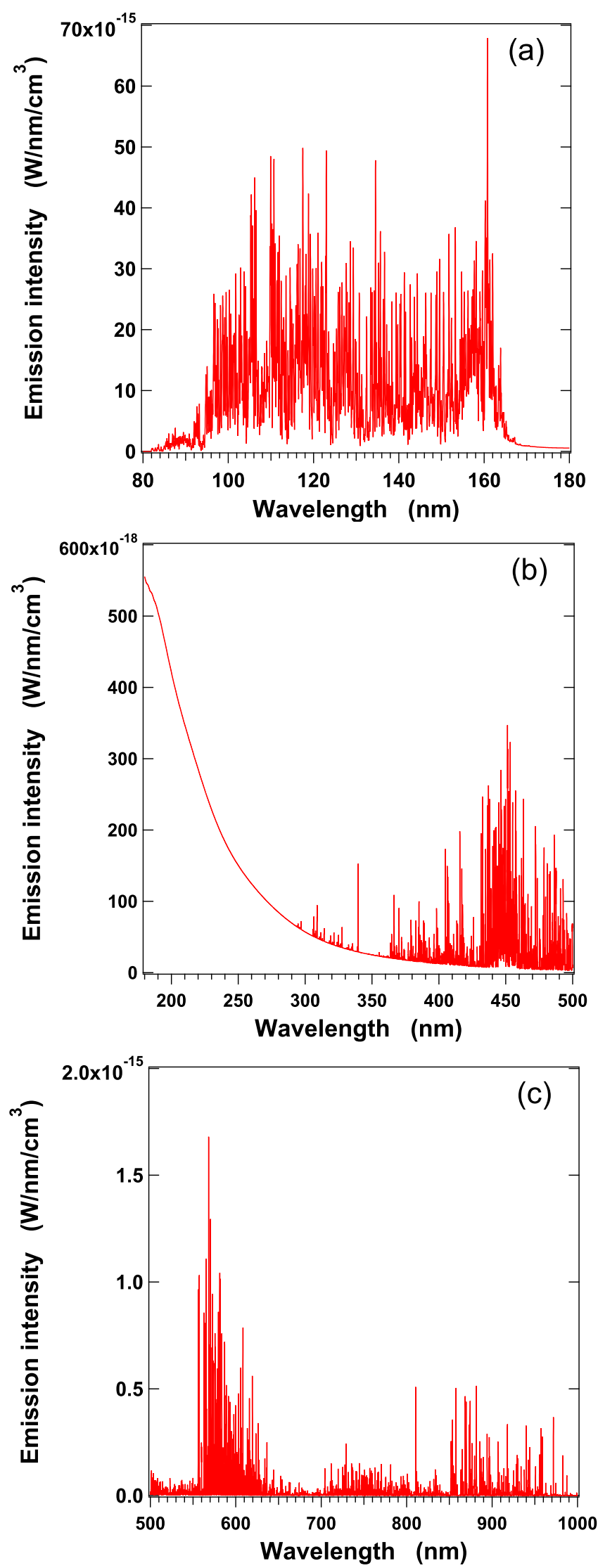

Figure 15. Emission spectra of molecular hydrogen at $t=10^{-8} \mathrm{~s}$ : (a) $80 \mathrm{~nm}-180 \mathrm{~nm}$; (b) $180 \mathrm{~nm}-500 \mathrm{~nm}$; (c) $500 \mathrm{~nm}-1000 \mathrm{~nm}$. 


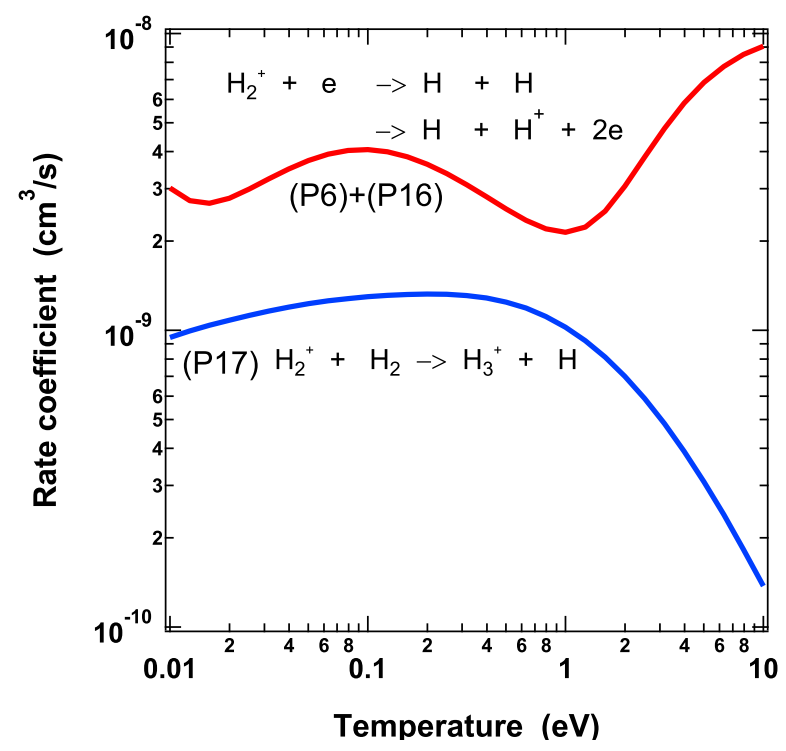

Figure 16. Rate coefficients for (P6) + (P16) and (P17) in Table 11, calculated using cross-section data from [74,75], respectively.

\section{Conclusions}

In this study, we constructed a rovibrationally-resolved collisional-radiative model of molecular hydrogen and applied it to a demo fusion detached divertor plasma. Using a time dependently solved population density distribution, the effective rate coefficients for MAR and the production of atomic hydrogen processes were calculated. The present model can provide rate coefficients for the purpose of tracing molecules in the rovibrational states in the $X^{1} \Sigma_{g}^{+}$as different species in neutral transport codes. Molecular spectra intensity is a function of $n_{\mathrm{H} 2}, n_{\mathrm{e}}, T_{\mathrm{e}}$ and the rovibrational population distribution of the $X^{1} \Sigma_{g}^{+}$. It was shown that the present $\mathrm{CR}$ model has the potential to determine these parameters from observed spectra. For this purpose, the population distribution of the excited electronic states should be calculated precisely by obtaining a reliable dataset for the electron impact excitation cross-sections between the electronic states. In this paper, we listed the used elementary process data. This will give some information on the necessary data. The present rovibrationally-resolved CR model will be utilized as a storage box for the rovibrationally-resolved elementary process data to be provided successively in the future. In addition to the above uses, the rovibrationally-resolved $\mathrm{H}_{2} \mathrm{CR}$ model can also be applied to the precise determination of the energy loss of projectile particles from collision with $\mathrm{H}_{2}$ in plasmas. We are constructing related models for isotope species $\mathrm{D}_{2}, \mathrm{~T}_{2}, \mathrm{HD}$ and DT that will be reported elsewhere.

Acknowledgments: This research was partially supported by a Grant-in-Aid for Scientific Research (C) 21540508 and 25400532 and (A) 16H02440 from the Japan Society for the Promotion of Science and by the NIFS Collaborative Research Program NIFS16KOAP031. We thank Hidekazu Takagi for useful comments and discussions. We also thank Yu Kambe, Toshimitsu Oda, Shinnosuke Kakura, Keita Nakane, Kazunari Taniguchi, Ryo Idei, Takanori Yashiro, Shinichi Hidaka and many other students of Shinshu University for their help in constructing the model.

Conflicts of Interest: The authors declare no conflict of interest.

\section{References}

1. Fujimoto, T. Plasma Spectroscopy; The International Series of Monographs on Physics, 123; Oxford University Press: Oxford, UK, 2004.

2. Sawada, K.; Fujimoto, T. Effective ionization and dissociation rate coefficients of molecular hydrogen in plasma. J. Appl. Phys. 1995, 78, 2913-2924. 
3. Greenland, P.T.; Reiter, D. The Role of Molecular Hydrogen in Plasma Recombination; Report JUEL-3258; Forschungszentrum: Jülich, Germany, 1996.

4. Wündrlich, D.; Fantz, U. A Collisional-Radiative Model for $\mathrm{H}_{2}$ and $\mathrm{H}$ : Extensions and Applications; Report IPP 10/18; Max-Planck-Institut fuer Plasmaphysik: Garching, Germany, 2001.

5. Reiter, D. Progress in two-dimensional plasma edge modelling. J. Nucl. Mater. 1992, 196-198, 80-89.

6. Krasheninnikov, S.I.; Pigarov, A.Yu.; Soboleva, T.K.; Sigmar, D.J. Plasma-neutral gas interaction in a tokamak divertor: Effects of hydrogen molecules and plasma recombination. J. Nucl. Mater. 1997, 241-243, $283-287$.

7. Sawada, K.; Fujimoto, T. Effect of initial vibrational excitation of molecular hydrogen on molecular assisted recombination in divertor plasmas. Contrib. Plasma Phys. 2002, 42, 603-607.

8. CRP on Atomic and Molecular Data for State-Resolved Modelling of Hydrogen and Helium and Their Isotopes in Fusion Plasma (2011-2015). Available online: https://www-amdis.iaea.org/CRP/ HydrogenHelium/ (accessed on 30 June 2016).

9. Fantz, U.; Heger, B. Spectroscopic diagnostics of the vibrational population in the ground state of $\mathrm{H}_{2}$ and $\mathrm{D}_{2}$ molecules. Plasma Phys. Control. Fusion 1998, 40, 2023-2032.

10. Xiao, B.; Kado, S.; Kajita, S.; Yamasaki, D. Rovibrational distribution determination of $\mathrm{H}_{2}$ in low temperature plasmas by Fulcher- $\alpha$ band spectroscopy. Plasma Phys. Control. Fusion 2004, 46, 653-668.

11. Sawada, K.; Goto, M.; Ezumi, N. Spectroscopic diagnostic of helium-hydrogen rf plasma under the influence of radiation trapping. Plasma Fusion Res. 2010, 5, 1401010.

12. Hoshino, K.; Shimizu, K.; Asakura, N.; Takizuka, T.; Nakamura, M.; Tobita, K. Simulation study of an extended divertor leg for heat control in the SlimCS DEMO reactor. Contrib. Plasma Phys. 2012, 52, 550-554.

13. Hoshino, K.; Sawada, K.; Idei, R.; Tokunaga, S.; Asakura, N.; Shimizu, K.; Ohno, N. Photon trapping effects in DEMO divertor plasma. Contrib. Plasma Phys. 2016, 56, 657-662.

14. Fujimoto, T.; Miyachi, S.; Sawada, K. New density diagnostic method based on emission line intensity ratio of neutral hydrogen in an ionizing phase plasma. Nucl. Fusion 1988, 28, 1255-1263.

15. Sawada, K.; Fujimoto, T. Temporal relaxation of excited-level populations of atoms and ions in a plasma: Validity range of the quasi-steady-state solution of coupled rate equations. Phys. Rev. E 1994, 49, 5565-5573.

16. Herzberg, G. Molecular Spectra and Molecular Structure: Spectra of Diatomic Molecules, 2nd ed.; Krieger Publishing Co.: Malabar, FL, USA, 1989.

17. Sobolev, N.N. Electron-Excited Molecules in Nonequilibrium Plasma; Nova Science Publishers: Commack, NY, USA, 1989; pp. 121-173.

18. Crosswhite, H.M. Hydrogen Molecule Wavelength Tables of Gerhard Heinrich Dieke; John Wiley \& Sons Inc.: Hoboken, NJ, USA, 1972.

19. Lee, T.-G.; Balakrishnan, N.; Forrey, R.C.; Stancil, P.C.; Shaw, G.; Schultz, D.R.; Ferland, G.J. Rotational quenching rate coefficients for $\mathrm{H}_{2}$ in collisions with $\mathrm{H}_{2}$ from 2 to 10,000 K. Astrophys. J. 2008, 689, 1105-1111.

20. Bailly, D.; Salumbides, E.J.; Vervloet, M.; Ubachs, W. Accurate level energies in the $E F^{1} \Sigma_{g}^{+}, G K^{1} \Sigma_{g}^{+}, H^{1} \Sigma_{g}^{+}$, $B^{1} \Sigma_{u}^{+}, B^{1} \Sigma_{u}^{+}, D^{1} \Pi_{u}, I^{1} \Pi_{g}, J^{1} \Delta_{g}$ states of $\mathrm{H}_{2}$. Mol. Phys. 2010, 108, 827-846.

21. Miller, T.A.; Freund, R.S. Singlet-triplet anticrossings in $\mathrm{H}_{2}$. J. Chem. Phys. 1974, 61, 2160-2162.

22. Ross, S.C.; Jungen, C. Multichannel quantum-defect theory of $n=2$ and 3 gerade states in $\mathrm{H}_{2}$ : Rovibronic'energy levels. Phys. Rev. A 1994, 50, 4618-4628.

23. Wolniewicz, L. Nonadiabatic energies of the ground state of the hydrogen molecule. J. Chem. Phys. 1995, 103, 1792-1799.

24. Orlikowski, T.; Staszewska, G.; Wolniewicz, L. Long range adiabatic potentials and scattering lengths for the EF, e and $h$ states of the hydrogen molecule. Mol. Phys. 1999, 96, 1445-1448.

25. Dressler, K.; Wolniewicz, L. A. Experiment and theory of high resolution spectra of rovibronic molecular states. Improved theoretical ${ }^{1} \Sigma_{u}^{+}$and ${ }^{1} \Pi_{u}$ wavefunctions, energies, and transition moments for the $\mathrm{H}_{2}$ molecule. Ber. Bunsenges. Phys. Chem. 1995, 99, 246-250.

26. Staszewska, G.; Wolniewicz, L. Adiabatic energies of excited ${ }^{1} \Sigma_{u}$ states of the hydrogen molecule. J. Mol. Spectrosc. 2002, 212, 208-212.

27. Wolniewicz, L.; Staszewska, G. Excited ${ }^{1} \Pi_{u}$ states and the ${ }^{1} \Pi_{u} \rightarrow X^{1} \Sigma_{g}^{+}$transition moments of the hydrogen molecule. J. Mol. Spectrosc. 2003, 220, 45-51.

28. Wolniewicz, L. Adiabatic potentials of the lowest in ${ }^{1} \Pi_{g}$ and ${ }^{1,3} \Delta_{g}$ states of the hydrogen molecule. J. Mol. Spectrosc. 1995, 169, 329-340. 
29. Staszewska, G.; Wolniewicz, L. Transition moments among ${ }^{3} \Sigma$ and ${ }^{3} \Pi$ states of the $\mathrm{H}_{2}$ molecule. J. Mol. Spectrosc. 1999, 198, 416-420.

30. Ko?os, W.; Rychlewski, J. Double-minimum potential energy curves resulting from adiabatic effects: The $4 s^{3} \Sigma_{g}^{+}$and $4 d^{3} \Sigma_{g}^{+}$states of the hydrogen molecule. J. Mol. Spectrosc. 1996, 177, 146-153.

31. Sharp, T.E. Potential-energy curves for molecular hydrogen and its ions. At. Data Nucl. Data Tables 1971, 2, 119-169.

32. Rychlewski, J.; Komasa, J. Explicitly Correlated Wave Functions in Chemistry and Physics: Theory and Applications (Progress in Theoretical Chemistry and Physics); Rychlewski, J., Ed.; Kluwer Academic Publishers: Dordrecht, The Netherlands, 2004; pp. 91-147.

33. Killingbeck, J.P. Microcomputer Quantum Mechanics; CRC Press: Boca Raton, FL, USA, 1985.

34. Hansson, A.; Watson, J.K.G. A comment on Hönl-London factors. J. Mol. Spectrosc. 2005, 233, $169-173$.

35. Watson, J.K.G. Hönl-London factors for multiplet transitions in Hund's case a or b. J. Mol. Spectrosc. 2008, 252, 5-8.

36. Wolniewicz, L.; Staszewska, G. ${ }^{1} \Sigma_{u}^{+} \rightarrow X^{1} \Sigma_{g}^{+}$transition moments for the hydrogen molecule. J. Mol. Spectrosc. 2003, 217, 181-185.

37. Wolniewicz, L. Transition moments between ${ }^{1} \Delta_{g},{ }^{1} \Pi_{g}$, and ${ }^{1} \Pi_{u}$ states of the $\mathrm{H}_{2}$ molecule. J. Mol. Spectrosc. 1996, 180, 389-401.

38. Staszewska, G. Transition moments between $w^{3} \Pi_{g}$ state and the first three ${ }^{3} \Sigma_{u}$ and ${ }^{3} \Pi_{u}$ states of the hydrogen molecule. J. Phys. Chem. A 2001, 105, 2308-2311.

39. Astashkevich, S.A.; Lavrov, B.P. Tables of the mean lifetimes for excited electron-vibro-rotational states of isotopomers of diatomic hydrogen. 2008, arXiv:0812.4573v1.

40. Astashkevich, S.A.; Käning, M.; Käning, E.; Kokina, N.V.; Lavrov, B.P.; Ohl, A.; Röpcke, J. Radiative characteristics of $3 p \Sigma, \Pi ; 3 d \Pi^{-}, \Delta^{-}$states of $\mathrm{H}_{2}$ and determination of gas temperature of low pressure hydrogen containing plasmas J. Quant. Spectrosc. Radiat. Transf. 1996, 56, 725-751.

41. Abgrall, H.; Roueff, E.; Launay, F.; Roncin, J.-Y. The $B^{\prime 1} \Sigma_{u}^{+} \rightarrow X^{1} \Sigma_{g}^{+}$and $D^{1} \Pi_{u} \rightarrow X^{1} \Sigma_{g}^{+}$band systems of molecular hydrogen. Can. J. Phys. 1994, 72, 856-865.

42. Liu, X.; Ahmed, S.M.; Multari, R.A.; James, G.K.; Ajello, J.M. High-resolution electron-impact study of the far-ultraviolet emission spectrum of molecular hydrogen. Astrophys. J. Suppl. 1995, 101, 375-399.

43. MOLAT, Atomic and Molecular Data, l'Observatoire de Paris. Available online: https://molat.obspm.fr/ (accessed on 30 June 2016).

44. Day, R.L.; Anderson, R.J.; Sharpton, F.A. Radiative decay constants of the $\mathrm{H}_{2}$ Fulcher bands. J. Chem. Phys. 1978, 69, 5518-5520.

45. Stephens, T.L.; Dalgarno, A. Spontaneous radiative dissociation in molecular hydrogen. J. Quant. Spectrosc. Radiat. Transf. 1972, 12, 569-586.

46. Kwok, T.L.; Guberman, S.L.; Dalgarno, A.; Posen, A. Dipole moments and transition probabilities of the $a^{3} \Sigma_{g}^{+}-b \Sigma_{u}^{+}$system of molecular hydrogen. Phys. Rev A 1986, 34, 1962-1965.

47. Guberman, S.L.; Dalgarno, A. Dipole moments and transition probabilities of the ${ }^{3} \Pi_{g}-b^{3} \Sigma_{u}^{+},{ }^{3} \Pi_{u}-a^{3} \Sigma_{g}^{+}$, and ${ }^{3} \Pi_{g}-c^{3} \Pi_{u}$ systems of molecular hydrogen. Phys. Rev. A 1992, 45, 2784-2789.

48. Berg, Ch.; Ottinger, Ch. The forbidden predissociation of metastable $\mathrm{H}_{2}\left(c^{3} \Pi_{u}^{-}\right)$molecules studied by state specific lifetime measurements. J. Chem. Phys. 1994, 100, 8746-8754.

49. Celiberto, R.; Janev, R.K.; Laricchiuta, A.; Capitelli, M.; Wadehra, J.M.; Atems, D.E. Cross section data for electron-impact inelastic processes of vibrational excited molecules of hydrogen and its isotopes. At. Data Nucl. Data Tables 2001, 77, 161-213. (Cross section data for the electron impact excitation from $X^{1} \Sigma_{g}^{+}$to $B^{1} \Sigma_{u}^{+}$ and $C^{1} \Pi_{u}$ : private communication.)

50. Janev, R.K.; Reiter, D.; Samm, U. Collision Processes in Low-Temperature Hydrogen Plasmas. Available online: http://www.eirene.de/report_4105.pdf (accessed on 30 June 2016).

51. Miles, W.T.; Thompson, R.; Green, A.E.S. Electron-impact cross-sections and energy deposition in molecular hydrogen. J. Appl. Phys. 1972, 43, 678-686.

52. Möhlmann, G.R.; de Heer, F.J. Emission cross-sections of the $\mathrm{H}_{2}\left(3 p^{3} \Pi_{u} \rightarrow 2 s^{3} \Sigma_{g}^{+}\right)$transition for electron impact on $\mathrm{H}_{2}$. Chem. Phys. Lett. 1976, 43, 240-244.

53. Morrison, M.A.; Crompton, R.W.; Saha, B.C.; Petrovic, Z.L. Near-threshold rotational and vibrational excitation of $\mathrm{H}_{2}$ by electron impact: Theory and experiment. Aust. J. Phys. 1987, 40, 239-281. 
54. Horáček, J.; Č́́žek, M.; Houfek, K.; Kolorenč, P.; Domcke, W. Dissociative electron attachment and vibrational excitation of $\mathrm{H}_{2}$ by low-energy electrons: Calculations based on an improved nonlocal resonance model. II. Vibrational excitation. Phys. Rev. A 2006, 73, 022701.

55. Phelps, A.V. Cross sections and swarm coefficients for $\mathrm{H}^{+}, \mathrm{H}_{2}^{+}, \mathrm{H}_{3}^{+}, \mathrm{H}, \mathrm{H}_{2}$, and $\mathrm{H}^{-}$in $\mathrm{H}_{2}$ for energies from $0.1 \mathrm{eV}$ to $10 \mathrm{keV}$. J. Phys. Chem. Data 1990, 19, 653-675.

56. Ralchenko, Yu.V.; Janev, R.K.; Kato, T.; Fursa, D.V.; Bray, I.; de Heer, F.J. Cross Section Database for Collision Processes of Helium Atom with Charged Particles. 1. Electron Impact Processes; NIFS-DATA-59; NIFS: Toki, Japan, 2000.

57. Sartori, C.S.; da Paixão, F.J.; Lima, M.A.P. Superelastic cross-sections in $\mathrm{e}^{-}-\mathrm{H}_{2}$ scattering. Phys. Rev A 1997, $55,3243-3246$.

58. Sartori, C.S.; da Paixão, F.J.; Lima, M.A.P. Transitions between excited electronic states of $\mathrm{H}_{2}$ molecules by electron impact. Phys. Rev A 1998, 58, 2857-2863.

59. Laricchiuta, A.; Celiberto, R.; Janev, R.K. Electron-impact-induced allowed transitions between triplet states of $\mathrm{H}_{2}$. Phys. Rev A 2004, 69, 022706.

60. Celiberto, R.; Laricchiuta, A.; Janev, R.K. Electron-impact collision cross-sections involving $\mathrm{H}_{2}$ and $\mathrm{N}_{2}$ vibrationally and electronically excited molecules. J. Plasma Fusion Res. 2006, 7, 207-209.

61. Liu, X.; Shemansky, D.E. Ionization of molecular hydrogen. Astrophys. J. 2004, 614, 1132-1142.

62. Ichihara, A.; Iwamoto, O.; Janev, R.K. Cross sections for the reaction $\mathrm{H}^{+}+\mathrm{H}_{2}(\mathrm{v}=0-14) \rightarrow \mathrm{H}+\mathrm{H}_{2}^{+}$at low collision energies. J. Phys. B 2000, 33, 4747-4758.

63. Ichihara, A.; Iwamoto, O.; Yokoyama, K. Cross-sections for ion production in $\mathrm{H}^{+}+\mathrm{H}_{2}$ collisions calculated with the trajectory-surface-hopping method. At. Plasma-Mater. Interact. Data Fusion 2001, 9, 193-235.

64. Lane, N.F.; Geltman, S. Rotational excitation of diatomic molecules by slow electrons: Application to $\mathrm{H}_{2}$. Phys. Rev. 1967, 160, 53-66.

65. Horáček, J.; Čížek, M.; Houfek, K.; Kolorenč, P.; Pichl, L. Resonance contributions to low-energy electron collisions with molecular hydrogen. Nukleonika 2003, 48, 109-112.

66. Celiberto, R.; Janev, R.K.; Laporta, V.; Tennyson, J.; Wadehra, J.M. Electron-impact vibrational excitation of vibrationally excited $\mathrm{H}_{2}$ molecules involving the resonant ${ }^{2} \Sigma_{g}^{+}$Rydberg-excited electronic state. Phys. Rev. A 2013, 88, 062701.

67. Wrathmall, S.A.; Flower, D.R. The rovibrational excitation of $\mathrm{H}_{2}$ induced by H. J. Phys. B 2007, 40, 3221-3230.

68. Esposito, F.; Gorse, C.; Capitelli, M. Quasi-classical dynamics calculations and state-selected rate coefficients for $\mathrm{H}+\mathrm{H}_{2}(v, J) \rightarrow 3 \mathrm{H}$ processes: Application to the global dissociation rate under thermal conditions. Chem. Phys. Lett. 1999, 303, 636-640.

69. Horáček, J.; Houfek, K.; Čížek, M.; Murakami, I.; Kato, T. Rate Coefficients for Low-Energy Electron Dissociative Attachment to Molecular Hydrogen; NIFS-DATA-73; NIFS: Toki, Japan, 2003.

70. Celiberto, R.; Janev, R.K.; Wadehra, J.M.; Tennyson, J. Dissociative electron attachment to vibrationally excited $\mathrm{H}_{2}$ molecules involving the ${ }^{2} \Sigma_{g}^{+}$resonant Rydberg electronic state. Chem. Phys. 2012, 398, 206-213.

71. Sawada, K.; Eriguchi, K.; Fujimoto, T. Hydrogen-atom spectroscopy of the ionizing plasma containing molecular hydrogen: Line intensities and ionization rate. J. Appl. Phys. 1993, 73, 8122-8125.

72. Rabli, D.; Morrison, M.A. Importance of non-resonant scattering in low-energy dissociative electron attachment to molecular hydrogen. Phys. Rev. Lett. 2006, 97, 013201.

73. Janev, R.K.; Langer, W.D.; Evans, K.; Post, D.E. Elementary Processes in Hydrogen-Helium Plasmas: Cross Sections and Reaction Rate Coefficients; Springer: Heidelberg, Germany, 1987.

74. Takagi, H. Dissociative Recombination and Excitation of $\mathrm{H}_{2}^{+}, \mathrm{HD}^{+}$, and $\mathrm{D}_{2}^{+}$, with Electrons for Various Vibrational States. Phys. Scr. 2002, T96, 52-60.

75. Gentry, W.R.; McClure, D.J.; Douglass, C.H. Merged beams at Minnesota. Rev. Sci. Instrum. 1975, 46, 367-375.

76. Strasser, D.; Levin, J.; Pedersen, H.B.; Heber, O.; Wolf, A.; Schwalm, D.; Zajfman, D. Branching ratios in the dissociative recombination of polyatomic ions: The $\mathrm{H}_{3}^{+}$case. Phys. Rev. A 2001, 65, 010702.

77. Esposito, F.; Capitelli, M. Selective Vibrational Pumping of Molecular Hydrogen via Gas Phase Atomic Recombination. J. Phys. Chem. A 2009, 113, 15307-15314.

(C) 2016 by the authors; licensee MDPI, Basel, Switzerland. This article is an open access article distributed under the terms and conditions of the Creative Commons Attribution (CC-BY) license (http://creativecommons.org/licenses/by/4.0/). 